\title{
Exploration for novel inhibitors showing back-to-front approach against VEGFR-2 kinase domain (4AG8) employing molecular docking mechanism and molecular dynamics simulations
}

\author{
Shailima Rampogu, Ayoung Baek, Amir Zeb and Keun Woo Lee
}

\begin{abstract}
Background: Angiogenesis is a process of formation of new blood vessels and is an important criteria demonstrated by cancer cells. Over a period of time, these cancer cells infect the other parts of the healthy body by a process called progression. The objective of the present article is to identify a drug molecule that inhibits angiogenesis and progression.

Methods: In this pursuit, ligand based pharmacophore virtual screening was employed, generating a pharmacophore model, Hypo 1 consisting of four features. Furthermore, this Hypo 1 was validated recruiting, Fischer's randomization, test set method and decoy set method. Later, Hypo1 was allowed to screen databases such as Maybridge, Chembridge, Asinex and $\mathrm{NCl}$ and were further filtered by ADMET filters and Lipinski's Rule of Five. A total of 699 molecules that passed the above criteria, were challenged against 4AG8, an angiogenic drug target employing GOLD v5.2.2.

Results: The results rendered by molecular docking, DFT and the MD simulations showed only one molecule (Hit) obeyed the back-to-front approach. This molecule displayed a dock score of 89.77, involving the amino acids, Glu885 and Cys919, Asp1046, respectively and additionally formed several important hydrophobic interactions. Furthermore, the identified lead molecule showed interactions with key residues when challenged with CDK2 protein, 1URW.

Conclusion: The lead candidate showed several interactions with the crucial residues of both the targets. Furthermore, we speculate that the residues Cys919 and Leu83 are important in the development of dual inhibitor. Therefore, the identified lead molecule can act as a potential inhibitor for angiogenesis and progression.
\end{abstract}

Keywords: Angiogenesis, Progression, Back-to-front approach, VEGFR-2, MD simulations

\section{Background}

Initiation of tumour cell and its progression is a process which is performed by certain factors known as angiogenic factors [1]. Angiogenesis is a complex process during which the endothelial cells are involved in the generation of metallo-proteases, initiate cell migration, cell division,

\footnotetext{
* Correspondence: kwlee@gnu.ac.kr

Division of Applied Life Science (BK21 Plus Program), Systems and Synthetic Agrobiotech Center (SSAC), Plant Molecular Biology and Biotechnology Research Center (PMBBRC), Research Institute of Natural Science (RINS), Gyeongsang National University (GNU), 501 Jinju-daero, Jinju 52828, Republic of Korea
}

(c) The Author(s). 2018 Open Access This article is distributed under the terms of the Creative Commons Attribution 4.0 International License (http://creativecommons.org/licenses/by/4.0/), which permits unrestricted use, distribution, and reproduction in any medium, provided you give appropriate credit to the original author(s) and the source, provide a link to the Creative Commons license, and indicate if changes were made. The Creative Commons Public Domain Dedication waiver (http://creativecommons.org/publicdomain/zero/1.0/) applies to the data made available in this article, unless otherwise stated.

and further proliferation. Additionally, they are also responsible for the formation of the new cells [2]. More specifically, cancer has an ability of rapid cell growth and hence, it is evident that angiogenesis supports the cancer metastasis [1].It is therefore essential to identify the novel drug molecules, which could hinder angiogenesis.

Vascular Endothelial Growth Factors (VEGFs) demonstrate an essential role in angiogenesis and vasculogenesis [3] and therefore, portray to be an ideal drug targets for designing novel inhibitors. Typically, VEGFRs are the transmembrane proteins that are known to trigger 
angiogenesis through VEGF receptor signalling [4]. There are three different types of receptor tyrosine kinases (RTK) which display a high affinity towards VEGF, namely, VEGFR-1, VEGFR-2 and VEGFR-3, respectively. However, VEGFR-2 remains as the only protein kinase domain transmitting the angiogenic signals [5], while the VEGFR1 revealed a reduced activity than VEGFR-2 $[6,7]$ and VEGFR-3 exhibits its role in governing the embryonic angiogenesis [8]. Therefore, VEGFR-2 emerges as an ideal protein target to identify new drugs. Moreover, VEGFR-2 has a crucial role in rheumatoid arthritis [9] inflammation [10] porosis [11], metastasis [12] and ocular neovascularization [13]. Accordingly, identification of novel drugs against VEGFR-2 might also have a curative effect on the aforementioned diseases.

Depending upon the binding patterns, the tyrosine kinase inhibitors can be grouped into type I and type II [14]. Type I inhibitors interact with the Adenosine Triphosphate (ATP) binding site of the kinase in its active form [15] and thereby, displaying reduced selectivity, while in type II, the inhibitors bind to the ATP site along with the allosteric, hydrophobic site [16] and exhibits high selectivity. This interaction happens during the inactive state of the kinases [17]. The conserved triad Asp-Phe-Gly (DGF) governs the active and the inactive states of the kinase enzymes. Generally, DGF-in conformation was noticed in the active condition, whereas in the DFG-out is projected during the inactive state [17]. The simple architecture of VEGFR-2 active site comprises of the front and the back pocket. ATPbinding front pocket has two key residues associated with it, Glu917 and Cys919. The back hydrophobic pocket has Glu885 and Asp1046. Glu885 is seated on the $\alpha \mathrm{C}$ helix and the Asp1046 forms an important part of the triad $[18,19]$.

One of the highly significant characters of the cancer cells is being able to divide rapidly [20]. Cyclicdependent kinases (CDKs), are crucial enzymes and contribute at large towards this process and belong to the serine-threonine kinases subfamily [21]. These kinases have gained popularity for their role in cell division, differentiation, neuronal functions, transcription and apoptosis [22]. Of all the known CDKs, CDK1-CDK13, CDK2 is widely studied protein as it has a crucial role to be played during the cell cycle progression more specifically during the $\mathrm{G} 1$ to $\mathrm{S}$ phase transition $[22,23]$. However, this requires being associated with regulatory subunits such as cyclin A and E [23]. Cyclin A is complexed with CDK2 for the Sphase (Synthesis phase) progression while cyclin E is required during the retinoblastoma protein phosphorylation that provides the transition of G1/S (Gap1) $[23,24]$. Additionally, reports exists on the inhibition of CDK2 that can eventually lead to the killing of the cancerous cells by deregulation of E2F-1 activity [25]. Therefore, it will be beneficial to identify a small molecule that can act both on the angiogenesis and on progression. Such a type of inhibition was earlier reported by Antony et al. [26].

The objective of the present study is to identify a novel inhibitor that has a potential to interact both with the VEGFR protein and with the CDK2 protein. In the present investigation, an attempt was made to screen inhibitors that bind more precisely in the back-to-front fashion of the target protein. This approach has been proven extremely successful with respect to certain kinases [27-29].

Although, both the proteins share a very little homology, they exhibit structural similarity within the ATPbinding region. Accordingly, the current study tries to exploit these important features in identifying the novel dual drug/inhibitor. The investigation proceeds with the initial identification of VEGFR-2 inhibitors and then challenging them with the CDK2 protein.

\section{Methods}

\section{Preparation of the dataset}

A systematic literature survey was conducted to extract the dataset for VEGFR-2 inhibitors [30]. From the obtained information of 63 diverse compounds [31-39], 24 structurally diverse compounds were referred to as training set and the remaining 39 were grouped into test set compounds. The training set compounds were employed to build the pharmacophore model while the test set was used to validate the same. The important criteria in choosing the training set compounds is the inclusion of the most active compounds into it such that they impart the most reliable information pertaining to the generated pharmacophore model. Additionally, the training set compounds exhibited a wide range of, half maximal inhibitory concentration, $\mathrm{IC}_{50}$, spanning between 0.2 to $45,000 \mathrm{nmol} / \mathrm{L}$. Further, the compounds in the training set, (Fig. 1), were designated as most active $\left(\mathrm{IC}_{50}<250 \mathrm{nmol} / \mathrm{L},+++\right)$, moderately active $\left(250 \mathrm{nmol} / \mathrm{L} \leq \mathrm{IC}_{50}<5000 \mathrm{nmol} / \mathrm{L},++\right)$ and inactive $\left(\mathrm{IC}_{50} \geq 5000 \mathrm{nmol} / \mathrm{L},+\right)$, that was adapted based on the $\mathrm{IC}_{50}$ values. The same criteria were followed for the test set compounds. Their corresponding two dimensional (2D) structures were sketched using the ChemSketch (http://www.acdlabs.com/ resources/ freeware/ chemsketch/ Advanced Chemistry Development (ACD) Inc., Toronto, Canada) and were translated to their three dimensional (3D) structures adapting the Discovery Studio v4.5 (DS).

\section{Generation of the pharmacophore model}

In the recent times, pharmacophore modelling takes advantage of being one of the most reliable methods 


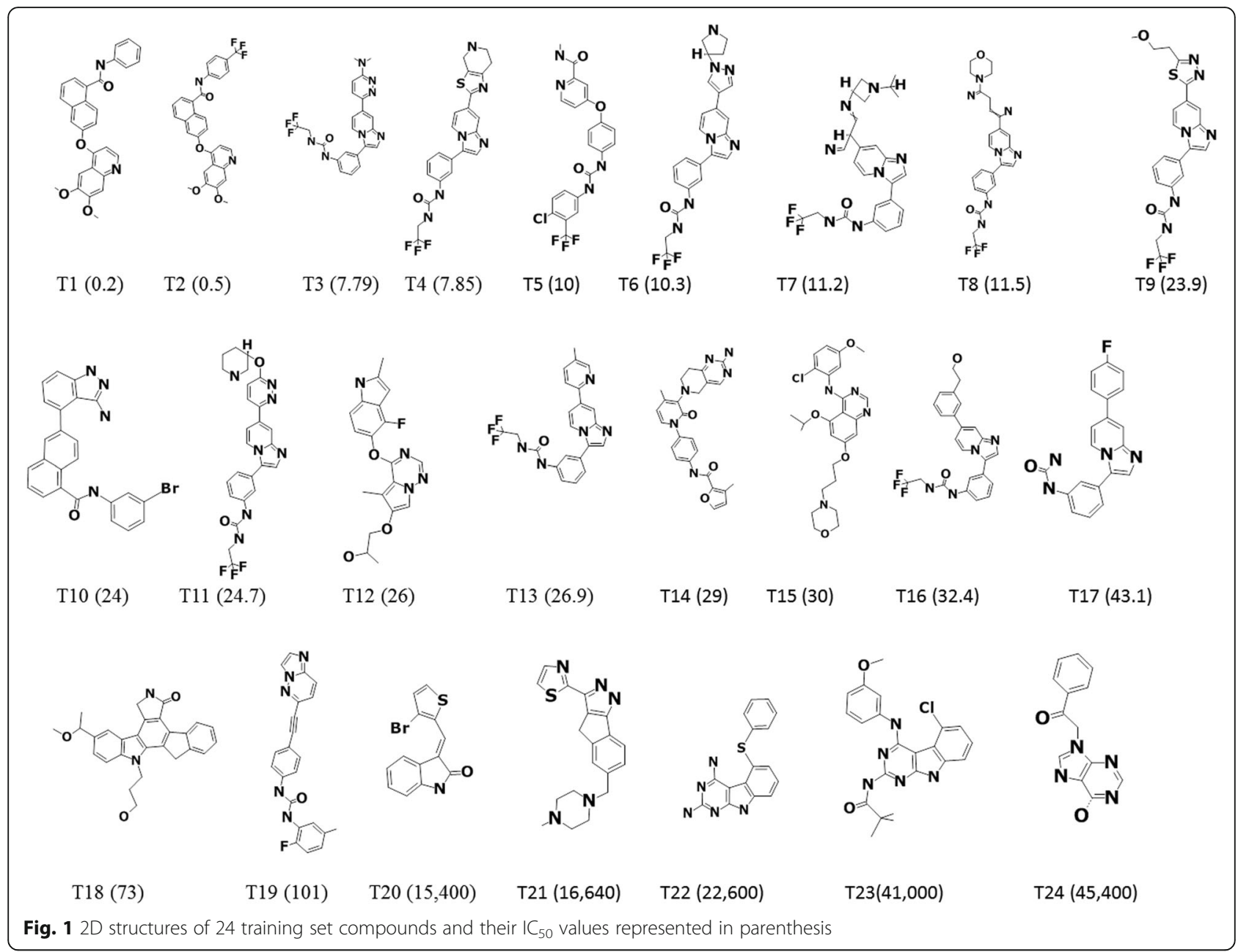

in identifying novel leads for different targets. For the present investigation, the three dimensional quantitative structure-activity relationship (3D-QSAR) based pharmacophore model was generated using the Catalyst HypoGen algorithm provided with the DS v4.5. This exploits the chemical features of the training set compounds and the conformation with the least energy were developed employing the BEST algorithm. In order to generate the best pharmacophore model, the energy and the uncertainty value were fixed at $20 \mathrm{kcal} /$ mol and 3, respectively [40]. Further, Feature Mapping protocol was employed for investigating into the chemical features and to recognize the common features present in the training set that could be essential in the pharmacophore generation. This protocol has an ability to construct pharmacophore features available with the training set compounds and further these identified features play a critical role in the generation of the model. Amongst the generated models, the best hypothesis was chosen based upon the Debnath's method [41].

\section{Validation of the generated pharmacophore model}

With an aim to determine the predictive ability and its capability to identify the active compounds from that of the inactives, the selected pharmacophore was subjected to validation recruiting three different approaches such as, Fischer's randomization, test set method, and the decoy set method. Fischer's randomization was carried out alongside the pharmacophore generation, which prompts random spreadsheets based upon the selected level of confidence. For the present investigation, the confidence level was chosen to be $95 \%$. The test and the decoy method of validations were conducted in order to understand if the generated pharmacophore was able to select the compounds in a similar manner as for the experimental activities. Ligand Pharmacophore Mapping protocol available on the DS was employed with Best Flexible algorithm. Test set was assembled with 39 structurally different compounds. The decoy set was instituted with a database of 710 compounds consisting of 15 active compounds. Following this, the enrichment 
factor (EF) and the goodness of fit score (GF) were computed using the formulae,

$$
\begin{aligned}
& G F=\left[\left(\frac{H a}{4 H t A}\right)(3 A+H t)\left(1-\frac{H t-H a}{D-A}\right)\right] \\
& E F=\frac{(H a X D)}{(H t X A)}
\end{aligned}
$$

Here, Ha represents the total number of active compounds, $\mathrm{Ht}$ refers to total hits redeemed from the database, A refers to the total number of active compounds in the database, and $\mathrm{D}$ denotes the total number of molecules in the database.

\section{Database screening for extracting the candidate compounds}

Validated pharmacophore was employed as the 3D query to screen the databases such as Maybridge, Asinex, Chembridge, and NCI to retrieve novel scaffolds against angiogenesis, if the chemical compounds mapped with all the chemical features present in the pharmacophore. In this pursuit, the Ligand Pharmacophore Mapping protocol was used with Best-Flexible options.

\section{Drug-likeness assessment}

Drug-likeness assessment was performed to the retrieved compounds from the databases so as to assess their biological activities. Accordingly, to judge the compound for strong pharmacokinetic properties, ADMET [42] and Lipinski's rule were applied. ADMET specifically evaluates if the compound can cross the Blood Brain Barrier (BBB), allowable solubility, good intestinal absorption with less toxicity. Therefore, the values 3,3 and 0 were fixed for $\mathrm{BBB}$, solubility and absorption, correspondingly and were computed adapting ADMET Descriptors module on the DS. Additionally, the Lipinski's Rule of 5 [43] was applied to the above filtered compounds to quantify if the prospective drug molecules could be well absorbed. This rule recommends a compound should have less than 10 hydrogen bond acceptors, less than 5 hydrogen bond donor groups having a molecular weight of less than $500 \mathrm{Da}$ with $\log p$ value of less than 5 with 10 rotatable bonds. All the compounds that satisfied the aforementioned criteria were forwarded for the docking studies.

\section{Molecular docking studies}

Challenging the potential screened lead molecules with the reliable drug target and to assess the degree of their binding affinities rendered in terms of the dock scores happens to be one of the most significant methodologies in drug discovery. Typically, this approach was deduced to assess the nature of the lead molecules in the active site and thereby its conformation. For the current study, Genetic Optimization for Ligand Docking v5.2.2 (GOLD) has been recruited [44, 45]. Target protein with the protein data bank (PDB) code, 4AG8, with high resolution of $1.95 \AA$, co-crystalled with axitinib was selected. Missing residues of the protein were rectified and all the hydrogen atoms were added, removing the water molecules [46]. Histidine tautomer orientations were placed in agreement with the crystal structure. The binding site of the protein was calculated for all the atoms that lie within the bound ligand around $15 \AA$. Furthermore, GOLD score was specified to understand the binding affinities between the ligand and the drug target, while the Chemscore was adapted for enumerating the rescoring function. Moreover, the GOLD score was initiated to generate 50 docking poses for each ligand and the reliable pose was selected based upon the highest dock score, molecular interactions and the hydrogen bonds that resulted between the ligand and the amino acid residues present at the active site of the protein molecule. Hereinafter, the most active compound in the training set was labeled as the reference compound.

The lead molecules identified after challenging against the 4AG8 would be further challenged with the CDK2 protein, 1URW, a potential target for cancer progression.

\section{Density functional theory}

DFT is one of the most dynamic methods adapted to calculate the electronic structure of matter and thus provides the most valuable information with respect to the selected inhibitors. DFT for the resultant docking molecules was performed using Becke, 3-parameter, LeeYang Parr (B3LYP) [47], available on the DS in order to evaluate their orbital energies such as highest occupied molecular orbitals (HOMO) and lowest unoccupied molecular orbitals (LUMO). HOMO refers to the electron donor and LUMO denotes the electron acceptor. DFT was executed together with the Hit compounds and the compounds from the training set.

\section{Molecular dynamics simulations}

To gain further insight into the protein-ligand interactions, the procured Hit compounds from the docking studies and the DFT were subjected to the Molecular Dynamics (MD) simulations along with the reference compound. The ligand topologies were generated utilizing the SwissParam [48-50], while the topologies of the protein were generated employing Chemistry at HARvard macromolecular Mechanics force-field (CHARMm ff) [51-54] implemented in Groningen Machine for Chemical Simulations (GROMACS) 5.0.6 [55]. Dodecahedron box was obtained and was solvated with three-site transferable intermolecular potential (TIP3P) water model followed by neutralizing the system with the counter ions. All the bad contacts were further removed by subjecting the system to 
pass through steepest descent algorithm at 10,000 steps with an upper limit of the force being lower than $1000 \mathrm{~kJ} / \mathrm{mol}$ [56]. Following this, the equilibration was conducted by Number of particles, Volume and Temperature (NVT) [57] and Number of particles, Pressure and Temperature (NPT) [58] at 100 ps at $300 \mathrm{k}$ and $100 \mathrm{ps}$ at a pressure of 1 bar maintained by Parrinello-Rahman barostat and allowing the movement of the counter ions and the water molecules, constraining the protein backbone. Linear Constraint Solver for Molecular Simulations (LINCS) [59] algorithm was used to restrain heavy atom bonds and their respective hydrogen atoms. Particle Mesh Ewald (PME) [60] was utilized to compute the long rage electrostatic interaction and a cut-off distance of $12 \AA$ was attributed for Coulombic and van der Waals interactions. MD simulations were performed for $30 \mathrm{~ns}$ storing the coordinate data for every 2 fs. Corresponding results were evaluated employing the Visula Molecular Dynamics (VMD) [61] and DS, respectively.

\section{Results}

HypoGen based pharmacophore model generation

Using the HypoGen algorithm provided with the DS, 24 training set compounds were employed to develop the pharmacophore model, (Figs. 1 and 2), which resulted in the generation of 10 hypotheses, Table 1 , upon the utilization of $3 D$ QSAR Pharmacophore Generation protocol available with the DS. The preferred features for the pharmacophore generation were hydrogen bond acceptor (HBA), hydrogen bond donor (HBD), hydrophobic (HyP), hydrophobic aliphatic (Hy-Ali) and ring aromatic (RA).

From the generated models, Hypo1 was chosen as the best pharmacophore model as it satisfied the Debnath's rules, which states that a good pharmacophore model should consists of high cost difference, good correlation, least RMSD and low cost values. The generated pharmacophore model composed of aromatic feature (RA), one hydrophobic aliphatic feature (Hy-Ali) and two hydrophobic (HyP) features.

Further, to evaluate the predictive ability of the Hypo1, it was allowed to examine the inhibitory activities of 24 training set compounds. The training set compounds were grouped into most active, moderately active and the least active compounds based upon their $\mathrm{IC}_{50}$ values as, $\left(\mathrm{IC}_{50}<250 \mathrm{nmol} / \mathrm{L},+++\right),(250 \mathrm{nmol} /$ $\left.\mathrm{L} \leq \mathrm{IC}_{50}<5000 \mathrm{nmol} / \mathrm{L},++\right)\left(\mathrm{IC}_{50} \geq 5000 \mathrm{nmol} / \mathrm{L},+\right)$, correspondingly. Hypo1 calculated the inhibitory activity values of the training set in accordance with the experimental values, Table 2. Furthermore, Hypo1 has successfully mapped to the most active compound and the most inactive compound, (Figs. 3 and 4).

\section{Validation of the pharmacophore model, Hypo1}

In order to assess the quality of the generated pharmacophore, it was subjected to a series of validations such as, Fischer's randomization method, test set method and the decoy test method.

\section{Fischer's randomization method}

To ascertain the statistical robustness of Hypo1, Fischer's randomization was performed that was run alongside the pharmacophore generation. A confidence level of $95 \%$ was selected which resulted in the formation of 19 spreadsheets. Thereafter, hypothesis significance was calculated employing the formula, $[1-(1+\mathrm{X}) / \mathrm{Y}] \mathrm{X} 100$. Herein, $\mathrm{X}$ denotes sum of hypothesis, while $\mathrm{Y}$ indicates the total HypoGen runs, both the initial and the random runs. This method takes the advantage of assessing the correlation

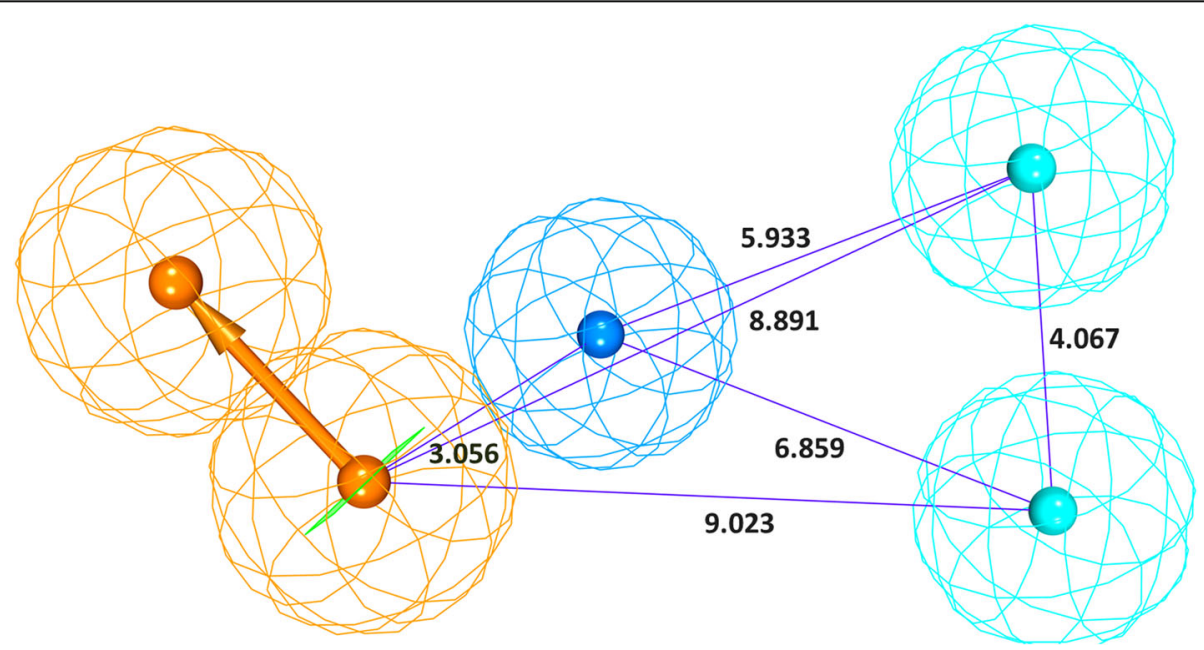

Fig. 2 Hypo1 pharmacophore model with its corresponding features and geometry, Aromatic Rings (brown), Hydrophobic Aliphatic (blue), 2 Hydrophobic (cyan) 
Table 1 Statistical data of the generated hypothesis employing HypoGen

\begin{tabular}{|c|c|c|c|c|c|c|}
\hline Hypo no & Total cost ${ }^{a}$ & Cost difference & $\mathrm{RMSD}^{\mathrm{b}}$ & Correlation & Features $^{c}$ & Max fit \\
\hline Hypo1 & 111.95 & 71.22 & 0.7 & 0.97 & HyAli, 2HyB,RA & 11.4 \\
\hline Hypo 2 & 113.31 & 69.86 & 0.7 & 0.96 & HyAli, 2HyB,RA & 11.5 \\
\hline Hypo 3 & 116.45 & 66.71 & 0.8 & 0.95 & HyAli,HyB,RA,HBA & 11.9 \\
\hline Hypo 4 & 116.47 & 66.69 & 1.0 & 0.94 & $\mathrm{HBA}, \mathrm{HBD} 2 \mathrm{HyB}$ & 10.7 \\
\hline Hypo 5 & 117.11 & 66.05 & 0.9 & 0.94 & HyAli,HyB,RA,HBA & 11.5 \\
\hline Hypo 6 & 119.51 & 63.65 & 1.0 & 0.93 & $\mathrm{HBA}, \mathrm{HBD}, 2 \mathrm{HyB}$ & 11.26 \\
\hline Нypo 7 & 119.52 & 63.65 & 0.9 & 0.95 & $\mathrm{HBA}, 2 \mathrm{HyB}, \mathrm{RA}$ & 12.65 \\
\hline Hypo 8 & 119.82 & 63.35 & 0.9 & 0.94 & $\mathrm{HBA}, \mathrm{Hy}-\mathrm{Ali}, \mathrm{HD}, \mathrm{RA}$ & 12.33 \\
\hline Hyро 9 & 119.94 & 63.23 & 1.2 & 0.91 & HBA, Hy-Ali,2HyB, RA & 11.98 \\
\hline Hypo10 & 120.52 & 62.65 & 1.1 & 0.91 & HBA,HBD,HY-Ali & 7.8 \\
\hline
\end{tabular}

${ }^{a}$ Cost difference between the null and the total cost. The null cost, the fixed cost and the configuration cost were found to be $183.177,101.77$ and 19.91 , respectively ${ }^{\mathrm{b}} \mathrm{RMSD}-$ Root Mean Square Deviation

'HyP- Hydrophobic, RA- Ring Aromatic, Hy-Ali-Hydrophobic Aliphatic, HBA-Hydrogen Bond Acceptor, HBD-Hydrogen Bond Donor

Table 2 Assessing the training set compound values for estimated and the experimental activities by Hypo 1

\begin{tabular}{|c|c|c|c|c|c|c|}
\hline Name & Fit value & $\begin{array}{l}\text { Experimental IC50 } \\
(\mathrm{nmol} / \mathrm{L})\end{array}$ & $\begin{array}{l}\text { Predicted IC } 50 \\
\text { (nmol/L) }\end{array}$ & Error $^{a}$ & Experimental scale & Predicted scale \\
\hline Molecule1 & 10.47 & 0.2 & 0.191 & -1.04 & +++ & +++ \\
\hline Molecule2 & 8.58 & 5.2 & 14.9 & 2.86 & +++ & +++ \\
\hline Molecule3 & 8.40351 & 7.79 & 22.7 & 2.91 & +++ & +++ \\
\hline Molecule4 & 8.4847 & 7.85 & 18.83 & 2.39 & +++ & +++ \\
\hline Molecule5 & 7.6927 & 10 & 116.68 & 11.66 & +++ & +++ \\
\hline Molecule6 & 8.4082 & 10.3 & 22.46 & 2.18 & +++ & +++ \\
\hline Molecule7 & 8.95463 & 11.2 & 6.38 & -1.75 & +++ & +++ \\
\hline Molecule8 & 8.44562 & 11.5 & 20.61 & 1.79 & +++ & +++ \\
\hline Molecule9 & 8.42254 & 23.9 & 21.73 & -1.09 & +++ & +++ \\
\hline Molecule10 & 8.004 & 24 & 56.97 & 2.37 & +++ & +++ \\
\hline Molecule11 & 8.36497 & 24.7 & 24.816 & 1 & +++ & +++ \\
\hline Molecule12 & 8.50932 & 26 & 17.79 & -1.46 & +++ & +++ \\
\hline Molecule13 & 8.20893 & 26.9 & 35.54 & 1.32 & +++ & +++ \\
\hline Molecule14 & 7.99567 & 29 & 58.08 & 2 & +++ & +++ \\
\hline Molecule15 & 8.48967 & 29.51 & 18.62 & -1.58 & +++ & +++ \\
\hline Molecule16 & 8.36485 & 32.4 & 24.82 & -1.3 & +++ & +++ \\
\hline Molecule17 & 8.19005 & 43.1 & 37.12 & -1.16 & +++ & +++ \\
\hline Molecule18 & 7.79069 & 73 & 93.11 & 1.27 & +++ & +++ \\
\hline Molecule19 & 8.47375 & 101 & 19.31 & -5.22 & +++ & +++ \\
\hline Molecule20 & 5.74562 & 15,400 & $10,329.50$ & -1.49 & + & + \\
\hline Molecule21 & 5.23885 & 16,600 & $33,178.10$ & 1.99 & + & + \\
\hline Molecule22 & 5.71617 & 22,600 & $11,054.20$ & -2.04 & + & + \\
\hline Molecule23 & 5.7456 & 41,200 & $10,330.10$ & -3.98 & + & + \\
\hline Molecule24 & 5.74648 & 45,000 & $10,309.20$ & -4.36 & + & + \\
\hline
\end{tabular}

${ }^{a}$ Error, ratio of the predicted activity (Pred IC50) to the experimental activity (Exp IC50) or its negative inverse if the ratio is $<1$ 


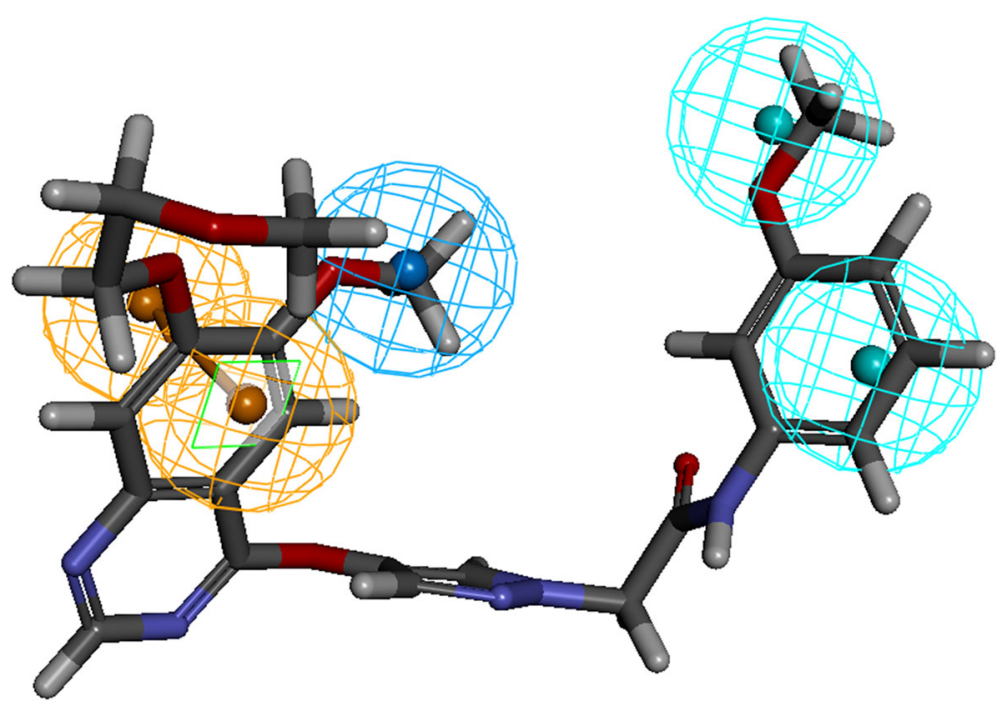

Fig. 3 Most active compound $\left(I_{50}=0.2\right)$ mapped to all the features

between the chemical structures and their corresponding biological activities, thereby quashing the chance correlation, and thus establishing that Hypo1 was not generated by chance. These results evidently state that the generated pharmacophore was of superior quality and thus, had least cost value as depicted in, Fig. 5.

\section{Test set validation}

Test set method of validation was conducted to examine the ability of the Hypo1 in recognizing the compounds other than the training set and thus to classify them in the same order of the activity range. 39 structurally diverse compounds were collected and were grouped in accordance with the training set. Subsequently, the correlation coefficient ( $r$ ) for the test set was computed as 0.95 , while that of the training set compounds was 0.96, (Fig. 6). Additionally, Hypo1 ably estimated the activities of the external compounds, however, overestimated three moderately active compounds as active compounds, (Additional file 1). The test set validation results are a clear indicative of the fact that Hypol can be recruited to classify the external compounds as well.

\section{Decoy set validation}

To further quantify the usefulness of the Hypo1, it was subjected to yet another validation process, called the decoy set method. This method was executed recruiting an external database. Accordingly, a database (D) of 710 molecules was instituted with an addition of 15 actives (A). Database screening was performed and as a result 17 Hit compounds were retrieved $(\mathrm{Ht})$ with 14 actives (Ha) in it. Further, the EF and the GF were computed to be 44.17 and 0.79 respectively, authenticating the goodness of the Hypo1, Table 3. Moreover, the percentage of

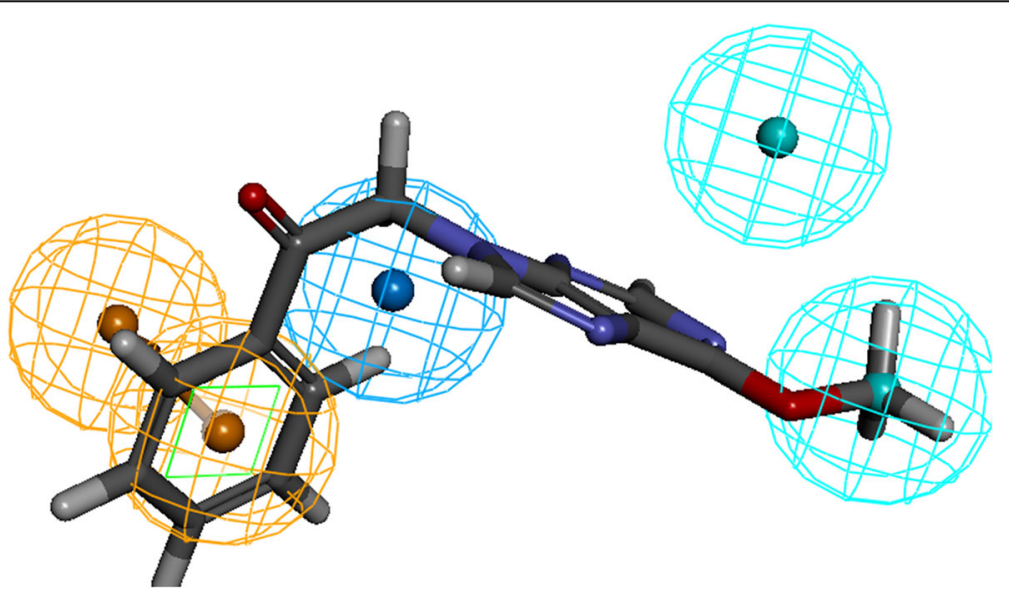

Fig. 4 Most inactive compound $\left(I C_{50}=45,000\right)$ aligned to only three features 


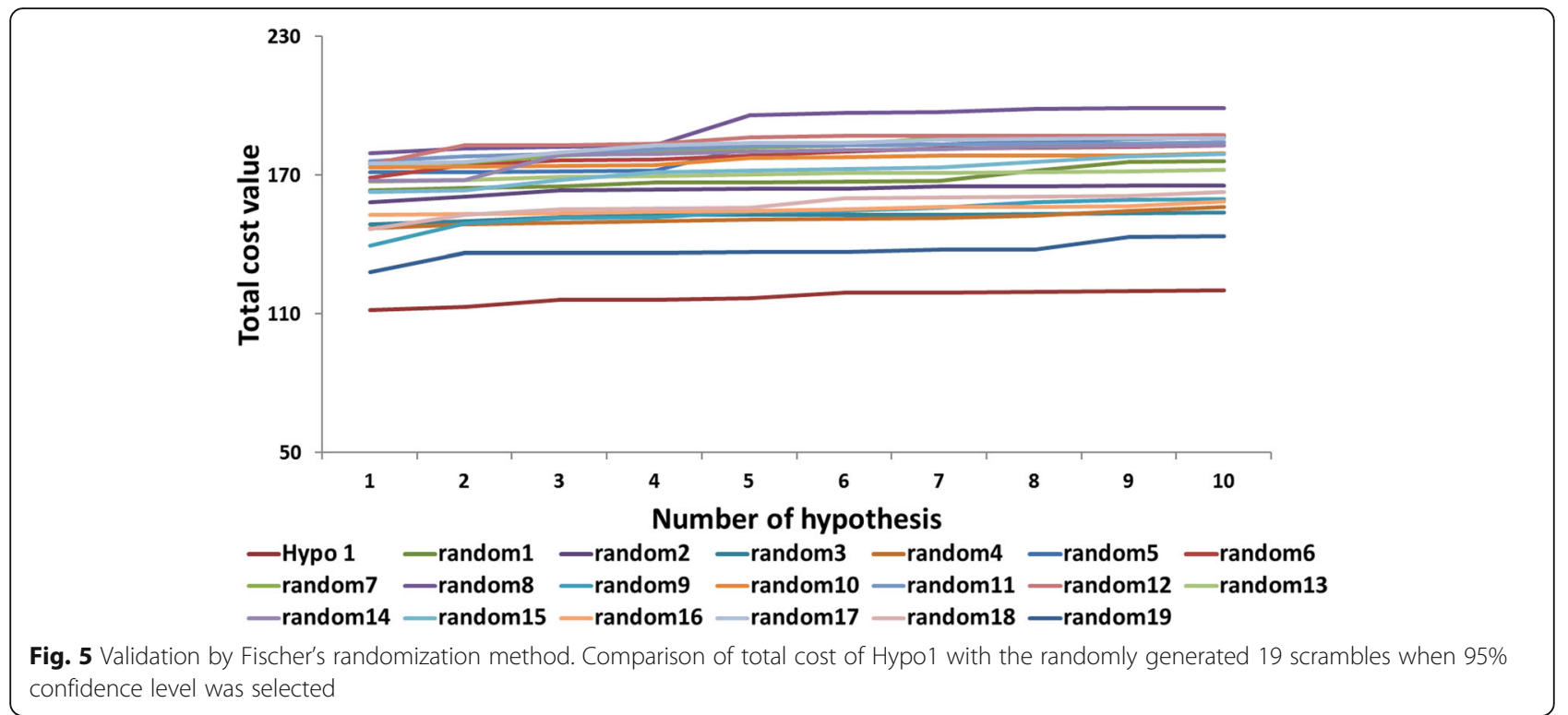

ratio of the actives was found to be 93 , pronouncing the high ability of Hypo1 in screening.

\section{Identifying the novel lead molecules through virtual screening}

Validated Hypo1 was employed to screen and retrieve the novel compounds against the VEGFR-2. The chemical features imbibed within the Hypo1 display an important role in identifying the new leads. Four databases, such as Chembridge, Maybridge, Asinex and NCI were selected for screening the dual inhibitors.
Accordingly, Hypo1 has successfully mapped with 12,080, $14,521,29,836$ and 29,660 compounds from the aforementioned databases. Following which, compounds with a greater fit value than 8 were proceeded to the Lipinski's Rule of Five and the ADMET studies. These two studies quantify the pharmacokinetics of a drug molecule and thus, is an essentiality for a drug molecule to qualify them. ADMET particularly computes BBB penetration, solubility, human intestinal absorption (HIA) solubility, plasma protein binding (PPB) and CytochromesP450 (CYPs) inhibition. The results were evaluated by setting

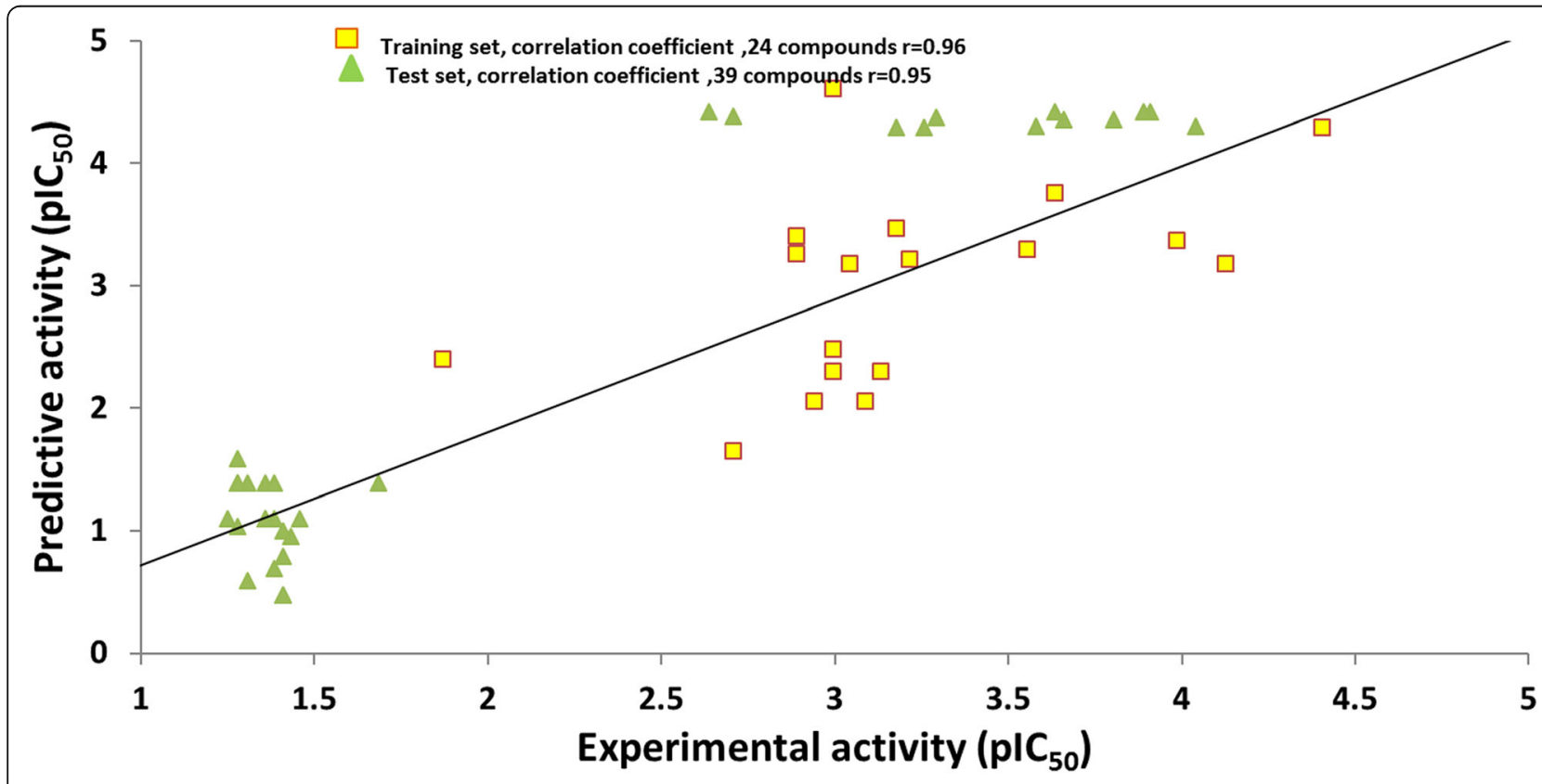

Fig. 6 Correlation prediction of Hypo1 between the test set and the training set compounds 
Table 3 Validation of Hypo1 by employing decoy set method

\begin{tabular}{lll}
\hline S. no & Parameters & Values \\
\hline 1 & Total number of molecules in database (D) & 710 \\
2 & Total number of actives in database (A) & 15 \\
3 & $\begin{array}{l}\text { Total number of hit molecules from } \\
\text { the database ( } \mathrm{Ht})\end{array}$ & 17 \\
4 & $\begin{array}{l}\text { Total number of active molecules in hit } \\
\text { list (Ha) }\end{array}$ & 14 \\
5 & \% Yield of active [(Ha/Ht) & 82 \\
7 & \% Ratio of actives [(Ha/A) X 100] & 93 \\
8 & Enrichment Factor (EF) & 44.17 \\
9 & False negatives (A-Ha) & 1 \\
10 & False Positives ( $\mathrm{Ht}-\mathrm{Ha})$ & 3 \\
\hline
\end{tabular}

"GH score between $0.6-0.8$ is regarded as a good score

the standard as 3,3 , and 0 for solubility, BBB and absorption, correspondingly. From the four databases, a total of 699 molecules were identified possessing the drug-like properties. The screened compounds along with the training set compounds were subjected to molecular docking analysis. The overall process of screening is represented pictorial form (Fig. 7).

\section{Molecular docking studies}

Genetic Optimization for Ligand Docking, (GOLD) v5.2.2 was recruited for performing the molecular docking assay. GOLD has an ability to conduct lead optimization and to determine the accurate binding pose analysis. Technically, GOLD operates by two scoring functions, Goldscore fitness, and the Chemscore.
Goldscore is a fitness parameter that is governed by four components, such as; protein-ligand hydrogen bond energy (external H-bond), protein-ligand van der Waals $(v d w)$ energy (external vdw), ligand internal vdw energy (internal vdw), ligand torsional strain energy (internal torsion), respectively. This score was optimized for understanding and predicting the position of the bound ligand. On the other hand, Chemscore was used for rescoring and further computes the total free energy change on the ligand binding. The aptness of the docking parameters were assessed by subjecting the cocrystal to dock into the selected active site. This generated a reasonable RMSD of $0.9 \AA$ between the docked pose and the co-crystal thus ensuring the accuracy of the GOLD parameters. Therefore, these parameters were considered for docking the screened compounds into the active site of the target, (Additional file 2).

Docking of the screened ligands and the training set compounds was initiated after specifying the radius of the active site around the co-crystal to 15 Angstrom $(\AA)$ followed by allowing the generation of 50 conformations for each ligand. All those compounds which fall in the selected radius were considered for the succeeding steps. The docking studies were initially executed with 4AG8, a potential drug target for angiogenesis. The final selected lead compounds after the DFT and the MD simulations were challenged with the CDK2 protein, 1URW. This strategy was conceived to identify a common drug molecule for both angiogenesis and progression.

The angiogenesis target for the present study was 4AG8, retrieved for the Protein Data Bank (PDB, http://www. rcsb.org/pdb/home/home.do). The reference molecule has

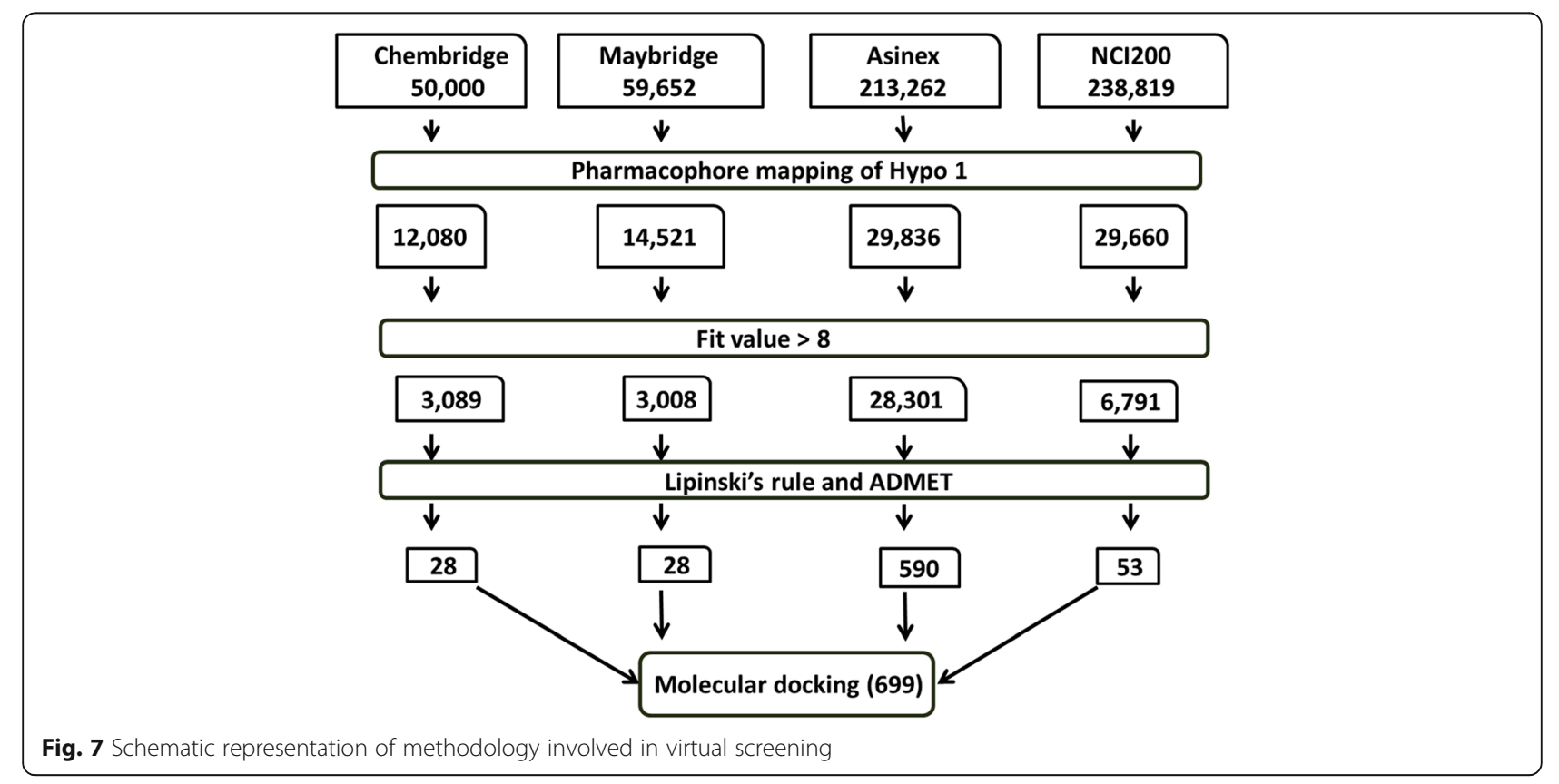


displayed a dock score of 89.77 and therefore, only those lead molecules whose scores were above the reference molecules were chosen for further investigation and thus, qualifying 18 screened molecules. These compounds were examined manually for the hydrogen bond interactions with the amino acids residues, Cys919, Glu917, Asp1046, and Glu885, respectively. Out of 18 screened lead molecules, only 11 exhibited the hydrogen bond interactions with the key residues. These 11 compounds, (Fig. 8), obeyed all the filters employed to identify an efficient lead candidate. These 11 molecules were subjected to the Density Functional Theory (DFT) analysis for further understanding their molecular orbital energies.

\section{Density functional theory studies}

Highest occupied molecular orbital (HOMO) and the lowest unoccupied molecular orbital (LUMO) that ascribe the DFT were computed with six training set compounds ( 2 active, 2 moderately active and 2 inactive compounds) and 11 screened compounds. Small gap obtained, reports a compound to be highly reactive, while the larger gap signifies the presence of low reactivity of the compound to that of the target protein [62]. Accordingly, compound 4 has been selected as it demonstrated the least gap, Table 4, obeys the back to front approach and maps to all the pharmacophore features, (Fig. 9). Hereafter, is compound was named Hit.

\section{Molecular dynamics simulations}

With an objective of assessing the binding stability of the final systems, the best conformations obtained from the docking were proceeded to MD simulations. Furthermore, MD simulation evaluates and delineates on their dynamic behaviour and with each other. The MD results were examined for the RMSD values, potential energy and the radius of gyration to assess the stability of the protein backbone. The RMSD values were found to be $1.0 \mathrm{~nm}$, and $0.8 \mathrm{~nm}$, respectively for the reference molecule and the Hit. Additionally, it can be observed that the RMSD profiles of the candidate compound was more stable than the reference, (Fig. 10). It was also noticed that the stability was attained after 20 ps for reference compound and while Hit 1 was stable after 25 ps, (Fig. 10). Furthermore, their stability was assessed by plotting the potential energy, (Fig. 11) and the radius of gyration, (Fig. 12). Both profiles have rendered results

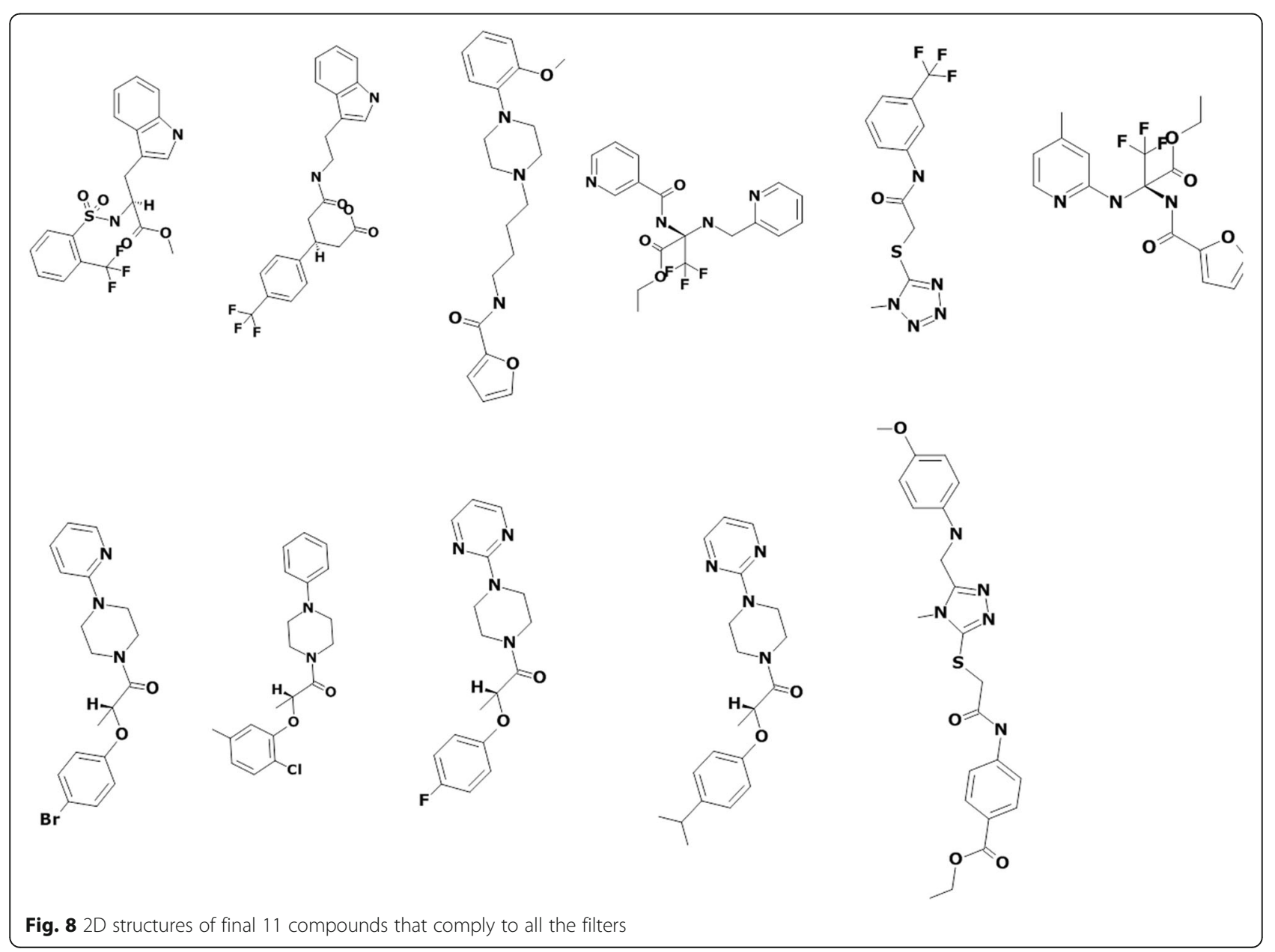


Table 4 Calculation of the orbital energy values of Hit compounds and training set compounds utilizing DFT. Only top four candidates are tabulated

\begin{tabular}{lllll}
\hline Name & $\mathrm{HOMO}(\mathrm{eV})$ & $\mathrm{LUMO}(\mathrm{eV})$ & $\Delta \mathrm{E}(\mathrm{eV})$ & $\mathrm{IC}_{50}$ \\
\hline Compound 1 & -0.17 & -0.02 & 0.14 & \\
Compound 2 & -0.18 & -0.06 & 0.11 & \\
Compound 3 & -0.19 & -0.07 & 0.12 & \\
Compound 4(Hit) & -0.15 & -0.08 & 0.07 & \\
T1 & -0.18 & -0.10 & 0.08 & 0.2 \\
T2 & -0.20 & -0.11 & 0.09 & 5.2 \\
T12 & -0.18 & -0.07 & 0.10 & 26 \\
T14 & -0.18 & -0.07 & 0.10 & 29 \\
T23 & -0.18 & -0.09 & 0.08 & 41,000 \\
T24 & -0.20 & -0.11 & 0.09 & 45,000 \\
\hline
\end{tabular}

that showed no abnormal behaviour throughout the simulation. Furthermore, the protein-Hit complex was found to be more compact as compared with the protein-reference complex, (Fig. 12). Additionally, from the Fig. 12 it can be understood that there were a few aberrations exhibited by the protein- reference complex and was stable after 25 ns. On the contrary, the proteinHit complex was found to be stable after $16 \mathrm{~ns}$, showing no aberrations thereafter.

The binding mode analysis was performed retrieving the representative structures from the last 3 ns trajectories. Upon superimposition, it was observed that the binding pattern of the Hit was similar with the reference compound, (Fig. 13). Further inspecting the molecular interactions of the reference, it was revealed that the reference has formed three hydrogen bonds demonstrated by two key residues, Cys919 and Asp1046, respectively and displaying an acceptable bond length, Table 5. The HN atom of the Cys919 interacted with N7 atom of the ligand and $\mathrm{NH}$ and $\mathrm{O}$ atoms of Asp1046 have joined with $\mathrm{O} 26$ and H62 of the ligand, (Fig. 14a). Leu840 has participated in the $\pi$-sigma interaction, while the residues, Ala866, Ala866, Val898, Leu889, and Leu1035 were found to interact through the $\pi$-alkyl bond, (Additional file 3 ). The Hit has interacted with the protein target with three hydrogen bonds represented one each by Glu885, Cys919 and Asp1046, respectively, (Fig. 14b). HN atom of Cys919 has involved with the ligand's $\mathrm{O} 14$ atom with a hydrogen bond length of $2.16 \AA$ and the OE2 atom of Glu885 joined to the OE2 atom of the ligand displaying a bond length of $2.09 \AA$, respectively. Additionally, Leu840, Val848, Lys868, Leu1035 have interacted involving with the $\pi-\pi$ bonds. Leu889 and Phe918 have interacted with the $\pi$-alkyl bond and thus make the ligand to be seated firmly within the active site groove, (Additional file 4). Moreover, from the large set of screened databases only one Hit was identified has the only retrieved compound that obeyed back-to-front pattern of binding and thus projecting itself as a unique lead compound, (Fig. 15). Correspondingly, it was observed that the Hit molecule has been positioned within the DFG motif comprising

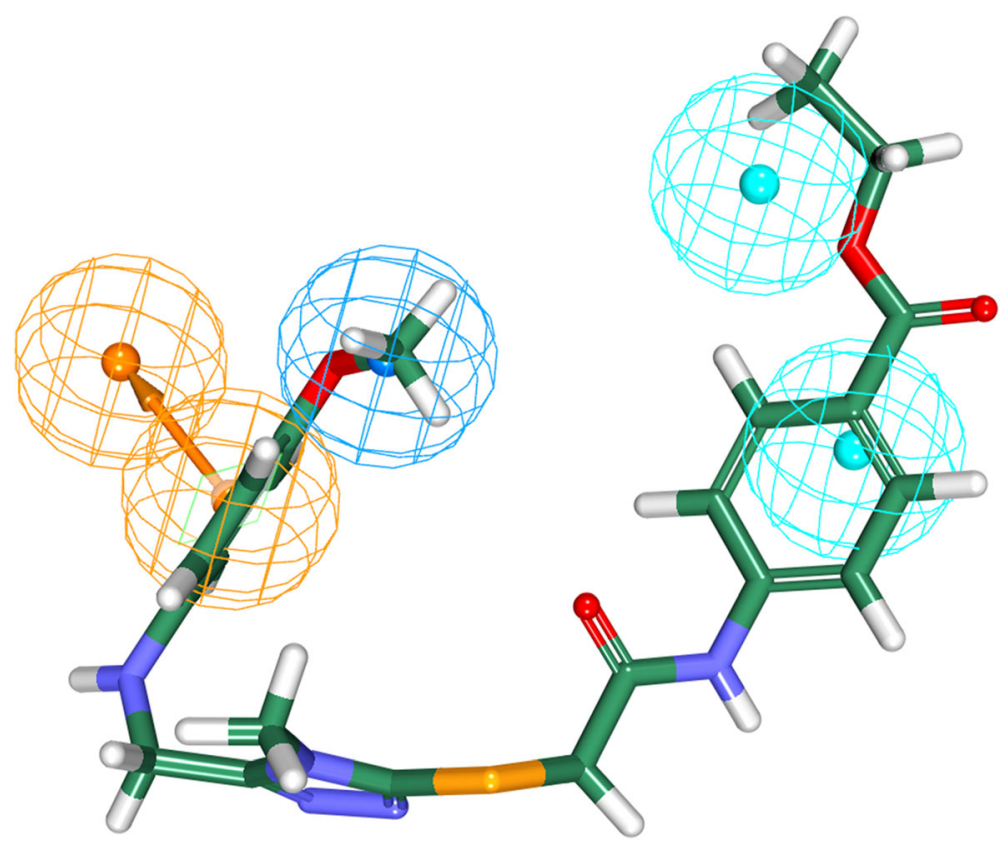

Fig. 9 Mapping of the Hit compound to all the features of the pharmacophore. The compound is found to be well aligned with all the pharmacophore features 


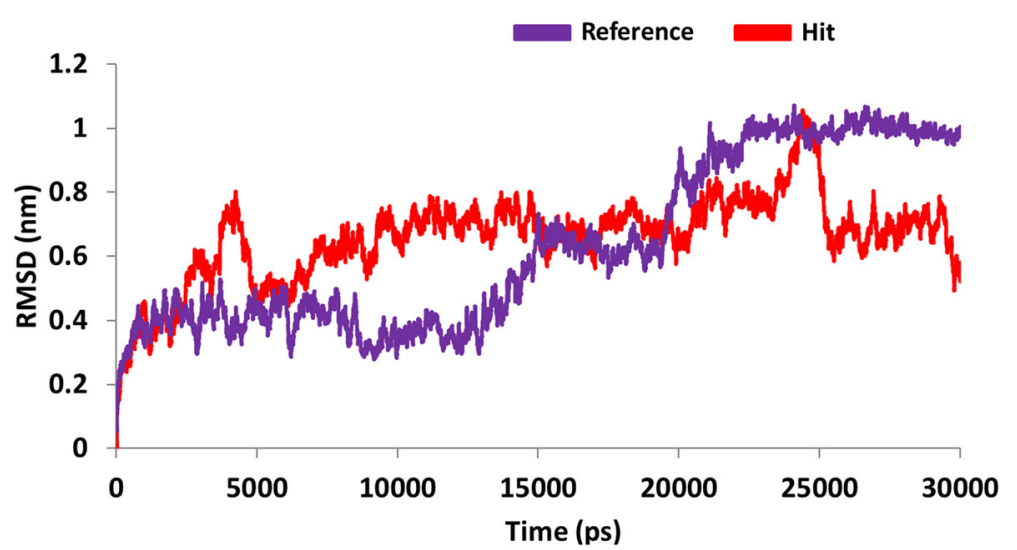

Fig. 10 Quantifying the overall stability of the backbone during 30 ps. Purlpe denotes the protein-reference complex and red denotes the protein-Hit complex

of Asp1046-Phe1047-Gly1048 residues sandwiched between Asp1046 and Glu885 on either side and hence crucial in conferring the reduction of angiogenesis. The Cys1045 has formed a $\pi$ - sulfur bond that makes the ligand to be placed firmly with the active site. A hydrophobic interaction have additionally concentrated towards the DFG motif of the activation loop and thereby, distorts the allosteric change of the motif and inhibits the kinase activity [5] and therefore the Hit may act as type II inhibitor. Further details of the interactions are tabulated in Table 5. Furthermore, to gain insight into and attain a proper reasoning on the nature of the hydrogen bonds in the active site, the hydrogen bonds were recorded throughout the simulation time. Consequently, it was noticed that the reference has produced an average of 0.7 hydrogen bonds, while the Hit has displayed a higher average of 1.7, (Fig. 16).
Challenging the identified lead molecule against CDK2 protein

After achieving the first objective, now the investigation proceeds to understand if the ligand is potential enough to act against cancer prognosis. Cyclin-Dependent Kinase 2(CDK2) has been attributed with certain cancer progressions, especially the oral cancer $[63,64]$. Progression refers to the advancement of the disease during the course of the time. For the current study the protein IURW, was retrieved from the protein data bank. This protein is in complex with imidazole [1,2-B] pyridazine inhibitor of $1.6 \AA$. The identified lead molecule form the above steps is now challenged with 1URW, such that a common drug could be identified.

All the steps, such as, protein preparation and ligand preparation were followed as per the aforementioned methods. The active site of the protein was plotted at

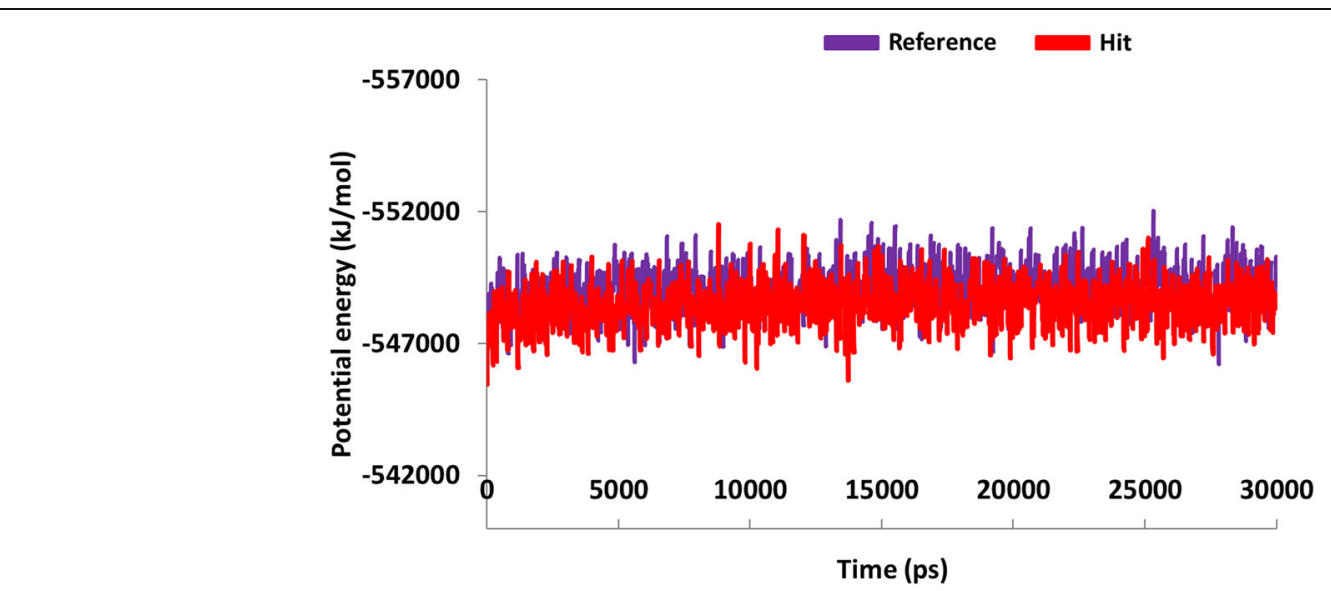

Fig. 11 Potential energy of protein-reference (purple) complex and protein-Hit complex (red). The plots show that both the complexes were well converged between $-547,000 \mathrm{~kJ} / \mathrm{mol}$ and $-551,000 \mathrm{~kJ} / \mathrm{mol}$ 


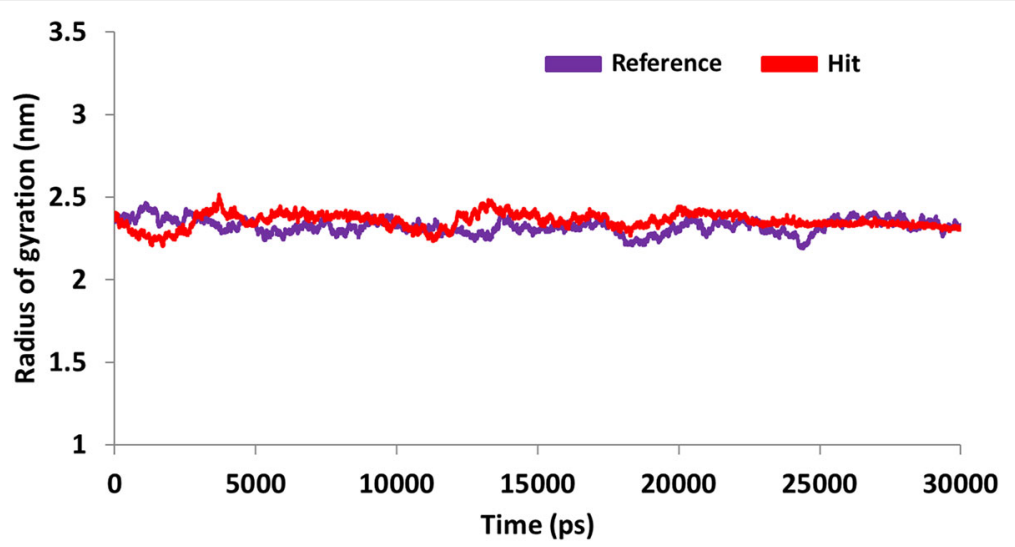

Fig. 12 Radius of gyration profiles protein-reference (purple) complex and protein-Hit complex (red). The protein-Hit complex was observed to be stably compact than the reference

$12 \AA$ around the inbuilt co-crystal. To ensure the suitability of the docking parameters, the co-crystal was initially docked and an acceptable RMSD of $1.75 \AA$ was generated, (Additional file 5) and the same parameters were considered for docking employing GOLD v5.2.2. The results were examined taking into consideration that the inhibitor lies within the selected active site sphere and forms a hydrogen bond with Leu83, Asp86 and Lys89. Amongst them, Leu83 is the core residue as reported earlier $[26,65]$. To authenticate the binding of the ligand with the active site residues, the results generated by the co-crystal docking were taken into consideration. The best pose generated was escalated to MD simulation studies to assess the protein backbone stability and were read as root mean square deviation (RMSD). Subsequent results demonstrated that the cocrystal, reference and the Hit have displayed stable RMSD values that exist below $0.25 \mathrm{~nm}$ throughout $30 \mathrm{~ns}$ run, (Fig. 17).

The representative structures from the last 3 ns were extracted and superimposed for further analysis. Upon superimposition of the co-crystal, reference and the Hit have demonstrated a similar type of binding mode, (Fig. 18). The dock results revealed that the co-crystal has formed four hydrogen bonds with residues namely Leu83, Asp86 and Lys89 demonstrating a dock score of 53.05, while the reference and Hit has displayed a score

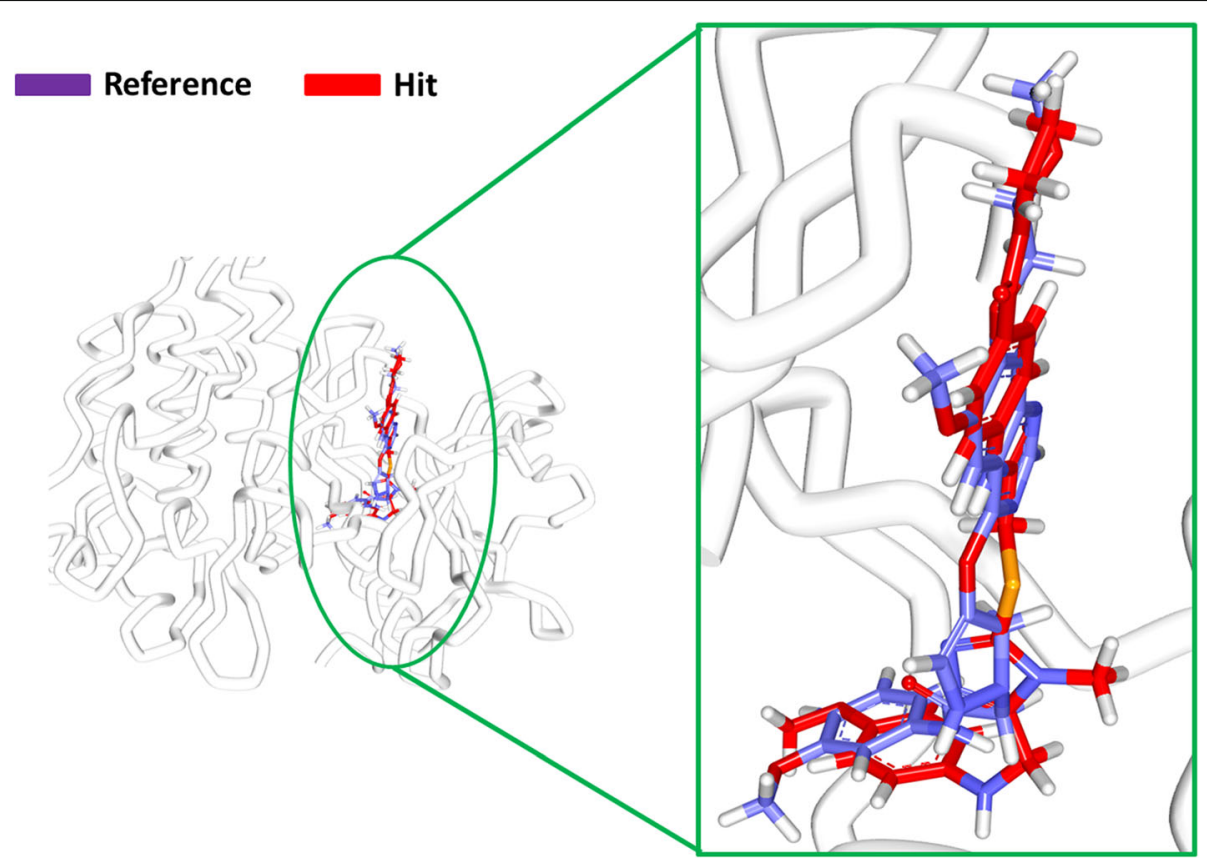

Fig. 13 Assessment of the binding mode patterns of the reference (purple) and the Hit (red). The Hit obeys the same binding pattern of the reference compound 
Table 5 Intermolecular interactions between the VEGFR-2 inhibitors

\begin{tabular}{|c|c|c|c|c|c|}
\hline \multirow{2}{*}{$\begin{array}{l}\text { Name of the } \\
\text { compound }\end{array}$} & \multicolumn{3}{|c|}{ Hydrogen bonds $<3 \AA$} & \multirow[t]{2}{*}{ van der Waals interactions } & \multirow[t]{2}{*}{$\pi-\pi$} \\
\hline & Residue atom & Ligand atom & Bond length & & \\
\hline Reference & $\begin{array}{l}\text { Cys919:HN } \\
\text { Asp1046:NH } \\
\text { Asp1046: O }\end{array}$ & $\begin{array}{l}\text { N7 } \\
\mathrm{O} 26 \\
\mathrm{H} 62\end{array}$ & $\begin{array}{l}2.2 \\
2.0 \\
2.0\end{array}$ & $\begin{array}{l}\text { Val848, Lys868, Glu885, Ile892, asn900, } \\
\text { Leu901, Lys920, Phe921, Gly922, Asn923, } \\
\text { Leu1019, leu1044, His1026 Phe1047 }\end{array}$ & $\begin{array}{l}\text { Leu840, Ala866, Ala866, } \\
\text { Val898, Leu889, Leu1035 }\end{array}$ \\
\hline Hit & $\begin{array}{l}\text { Glu885:OE2 } \\
\text { Cys919: O } \\
\text { Asp1046:HN }\end{array}$ & $\begin{array}{l}\mathrm{H} 36 \\
\mathrm{H} 35 \\
\mathrm{~N} 5\end{array}$ & $\begin{array}{l}1.88 \\
2.9 \\
2.3\end{array}$ & $\begin{array}{l}\text { Ala866, Glu850, Ile888, Ile892, Val898, } \\
\text { Val914, Lys920, Asn923, Gly922, Leu1019, } \\
\text { His1026, Ile1044, Phe1047 }\end{array}$ & $\begin{array}{l}\text { Leu840, Val848, Leu889, } \\
\text { Lys868, Phe918, Leu1035 }\end{array}$ \\
\hline
\end{tabular}

of 52.56 and 64.34. Additionally, a number of hydrophobic bonds have been generated in the form of van der Waals interactions and $\pi-\pi$ bonds as represented in Fig. 19a and Additional file 6. The reference compound on the other hand has rendered five hydrogen bonds one each by Glu12 and Gly16 and three bonds with Lys89. It was observed that only the Lys89 residue was noticed to participate as was seen in the crystal structure. Additionally, Glu12 formed a weak hydrogen bond of $3.0 \AA$ and displayed no interaction with Leu83. This could be because the compound is a specific VEGFR-2 inhibitor. However, it exerts its effect through the involvement of Lys89 residue, (Fig. 19b: Additional file 7). The retrieved Hit has displayed three hydrogen bonds involving two crucial residues, Leu83 and Lys89 and further has generated an acceptable bond length of $2.1 \AA$ and $2.4 \AA$, respectively, Fig. 19C. Additionally, it formed more number of hydrophobic interactions with the key resides, (Additional file 8 ). The details of the molecular interactions are tabulated in Table 6.

Furthermore, the binding pockets of both the targets were evaluated to understand their similarity and was assessed using PocketMatch (PM) [66] that was executed through three aspects (a) representation of each site as sorted lists of distances between chosen points, (b) alignment of two sets of distance lists and (c) choosing a scoring scheme for arriving at a final score. The similarity is read as PM score which ranges from 0 to 1 , where zero implies no similarity and 1 refers to complete similarity. The obtained results revealed the PM score to be 0.82 (0.6 or greater refers to almost similar). To further affirm the similarity of the active sites, the align structures protocol available on the DS was initiated and subsequently, the arrangement of the sequences, (Fig. 20) and the active site residues, (Fig. 21: Additional file 9) were evaluated. It was interesting to note that the majority of the key residues were conserved besides; the key residues Cys919 and Leu83 were complementary with each other and were instrumental in rendering the inhibitory activity in the corresponding drug targets. These findings further reinforce that they share similar binding pocket facilitating the use of common drug.

\section{Discussion}

Receptor tyrosine kinases (RTKs) are a class of proteins that play a crucial role in cell proliferation, differentiation,

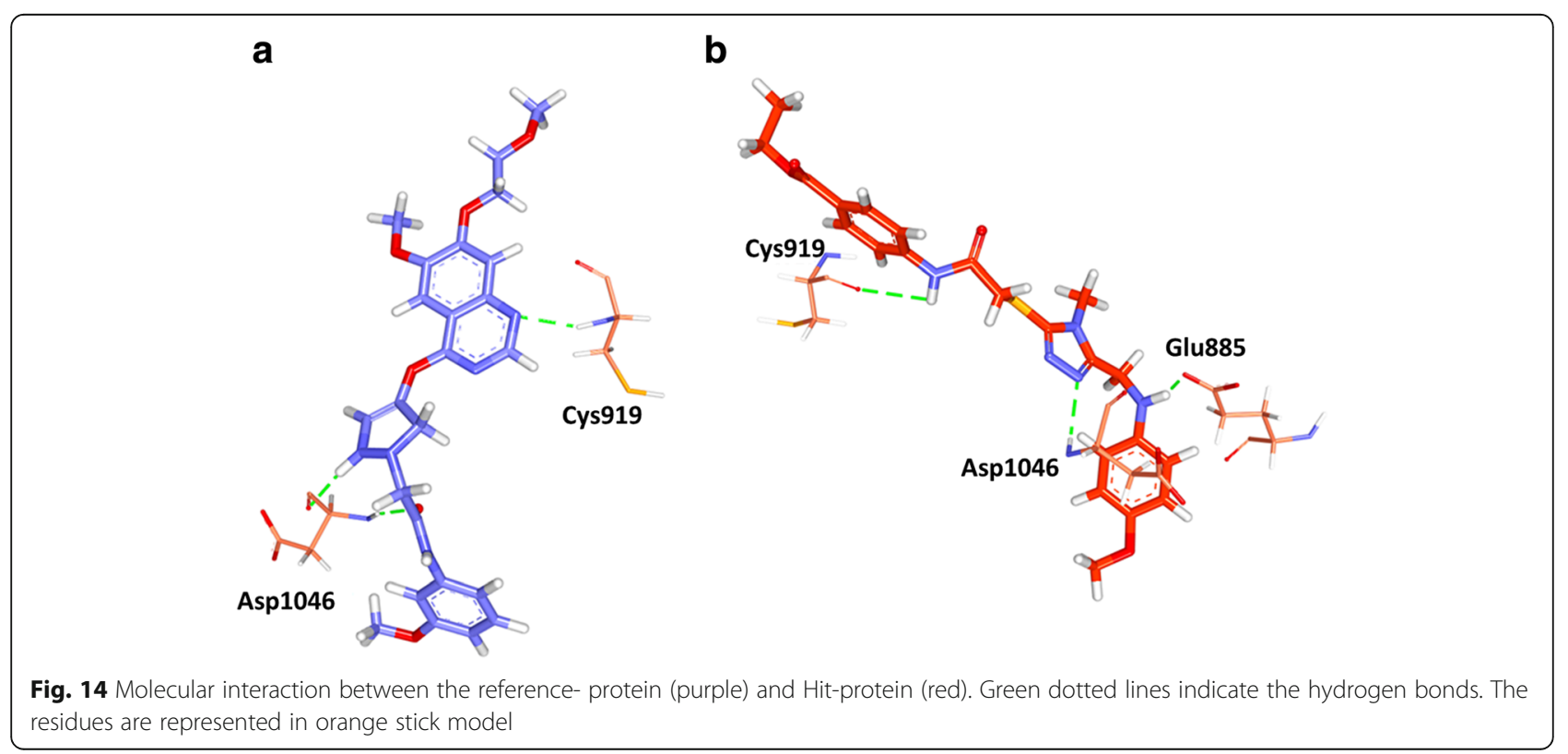




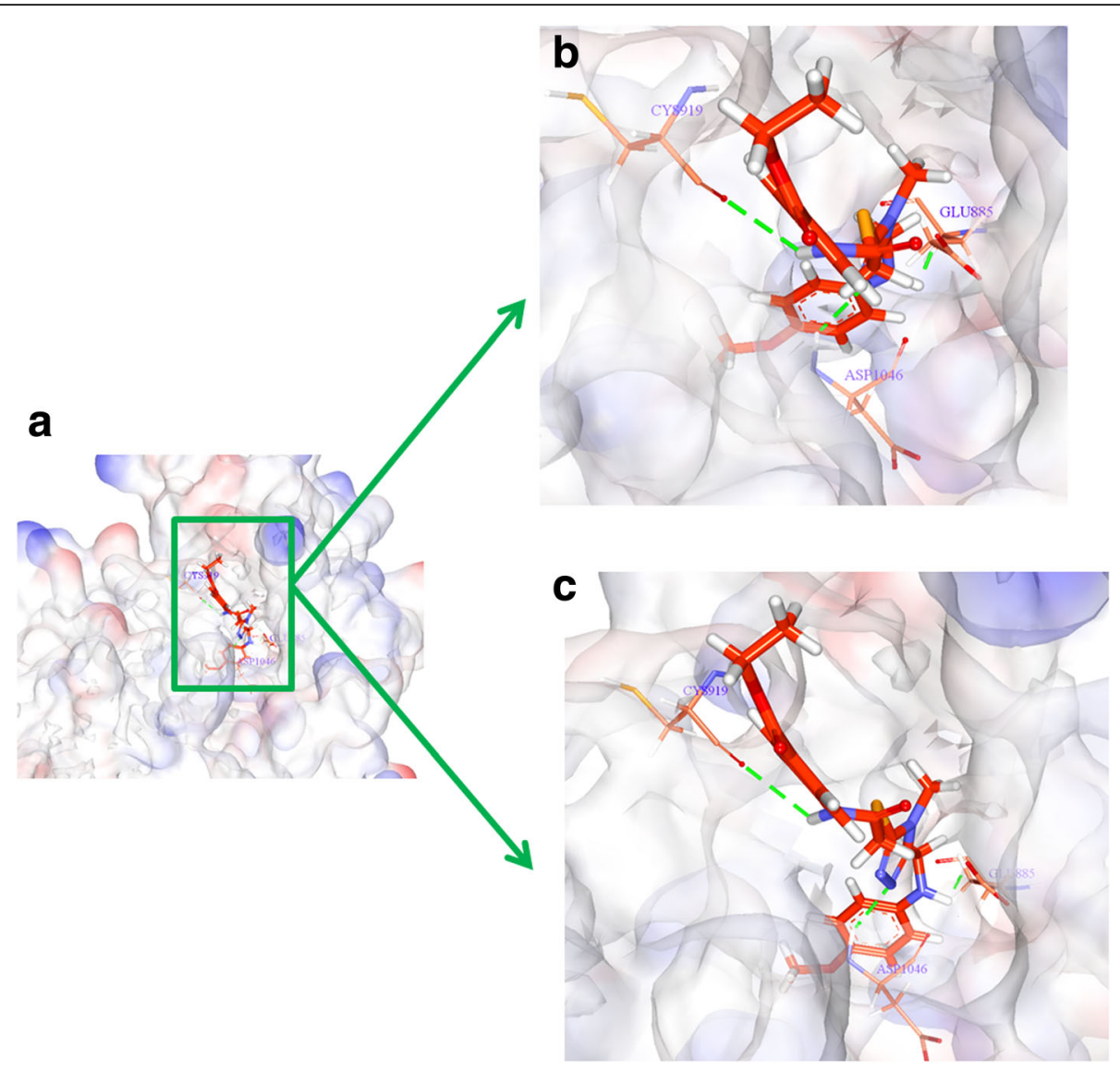

Fig. 15 Back-to-front pattern of binding. a) Depiction of the presence of Hit in the active site pocket in back-to-front fashion. A clear enlarged cavity is represented in b and c. The ligand was found to be seated firmly with Cys919 from the top and is sandwiched with Glu885 and Asp1046

metabolism and cell growth and survival. Among them, vascular endothelial growth factor receptor-2 (VEGFR-2) is important in angiogenic regulation and hence is a major target for repressing the cancer growth and metastasis [67-70] . Additionally, the uncontrolled development of these cancerous cells is the main reason for metastasis. Therefore, in the current study we aimed at identifying a small molecule from the large datasets that could selectively inhibit both the process, thus leading to suppression of cancer. Accordingly, only one lead

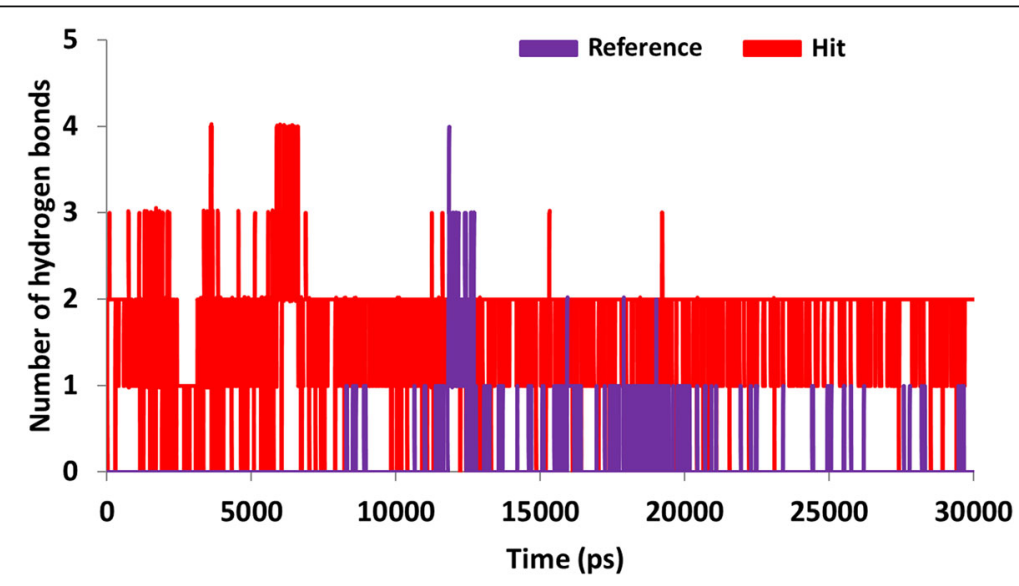

Fig. 16 Estimation of the hydrogen bond interactions during the whole simulations. The refrence is represented in purple and red denotes the Hit. The reference has produced lower number of hydrogen bonds and predominantly seen during 11,000 ps $\sim 20,000$ ps. The Hit has shown regular bonds throughout the simulations 


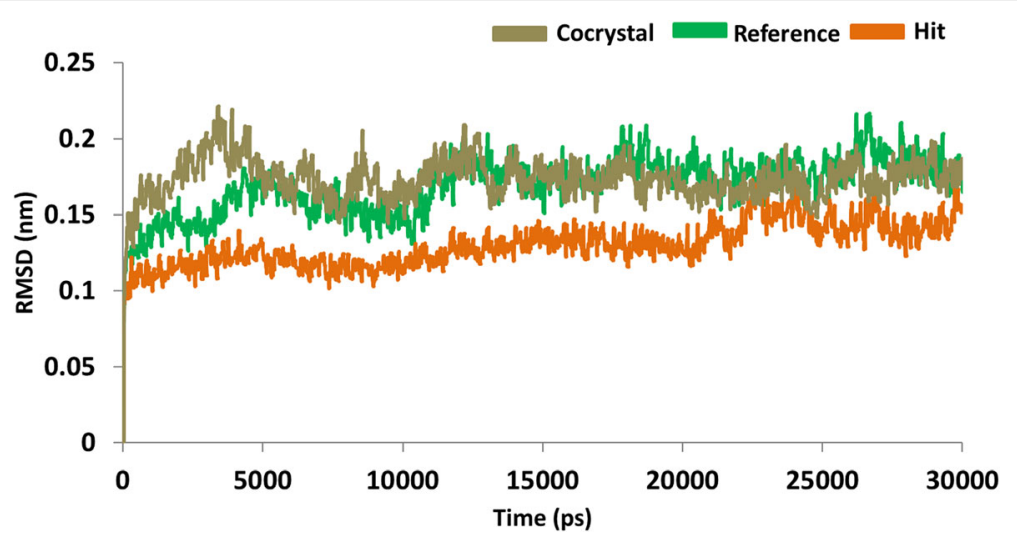

Fig. 17 The RMSD backbone stability of the three systems during 30 ns run

candidate has been identified that binds in back-tofront approach with the VEGFR-2 and also inhibits the CDK2. The identified inhibitor successfully binds to the ATP site (front pocket) and also extends towards the hydrophobic site (back pocket) and hence categorizes itself has type -II inhibitor. Cys919 located at the front pocket holds firmly the ligand molecule by $\mathrm{HN}$ atom and facilitates the bulky benzene ring to be accommodated within the active site with a slight tilt formed at the tip of the ligand comprised of $\mathrm{CH}_{3}$ group, (Fig. 15b). The back pocket on the other end was observed to be held tightly with two hydrogen bonds and several hydrophobic bonds, (Fig. 15c: Additional file 4). The pentane ring of the ligand that is located at the ligands center was seen to be held with two hydrophobic bonds produced by Val848 and Lys 868 besides displaying a hydrogen bond with Asp1046.

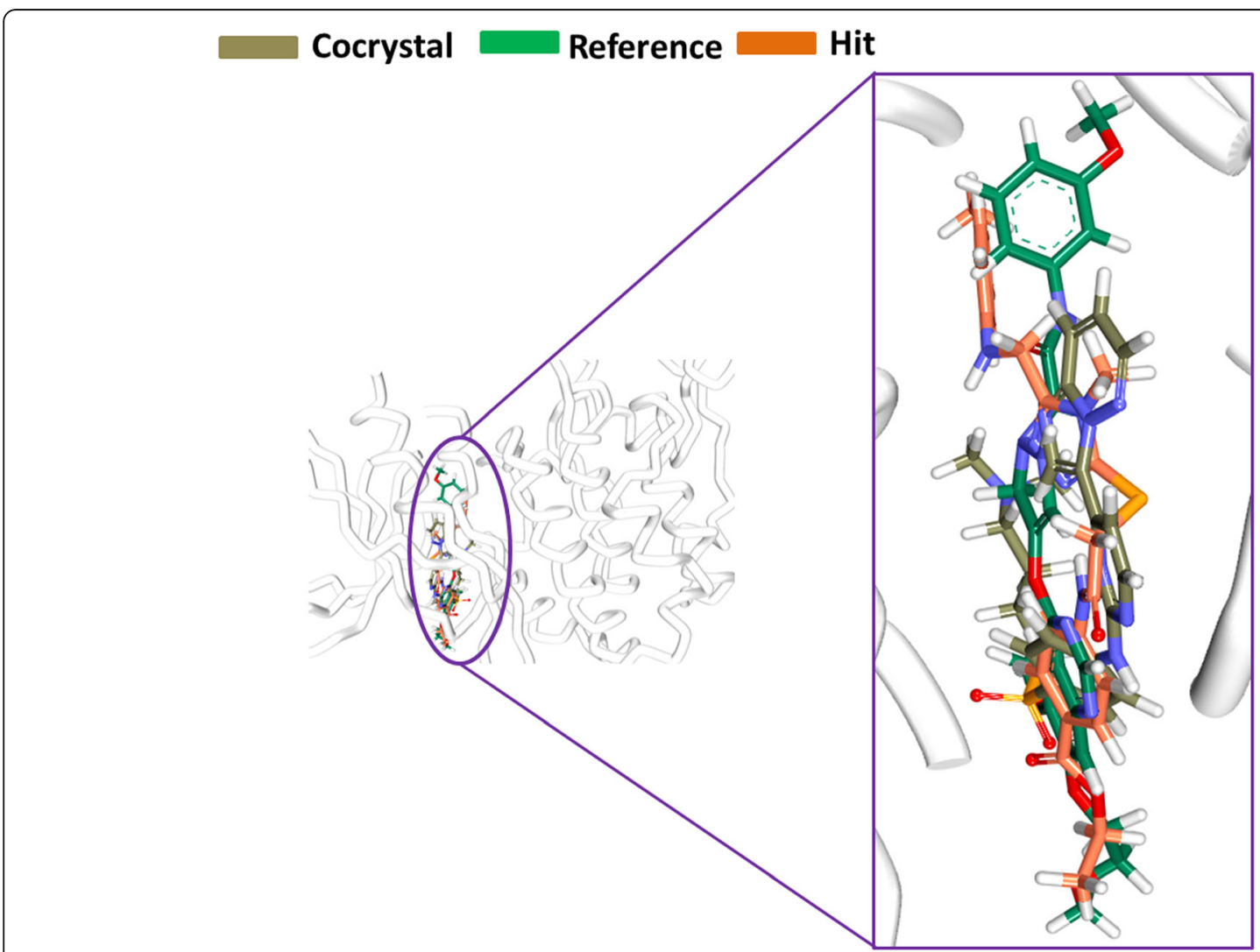

Fig. 18 Binding mode assessment of compounds. The co-crystal is represented in gray, reference is denoted in green and the Hit in orange. All the three follow the same pattern 

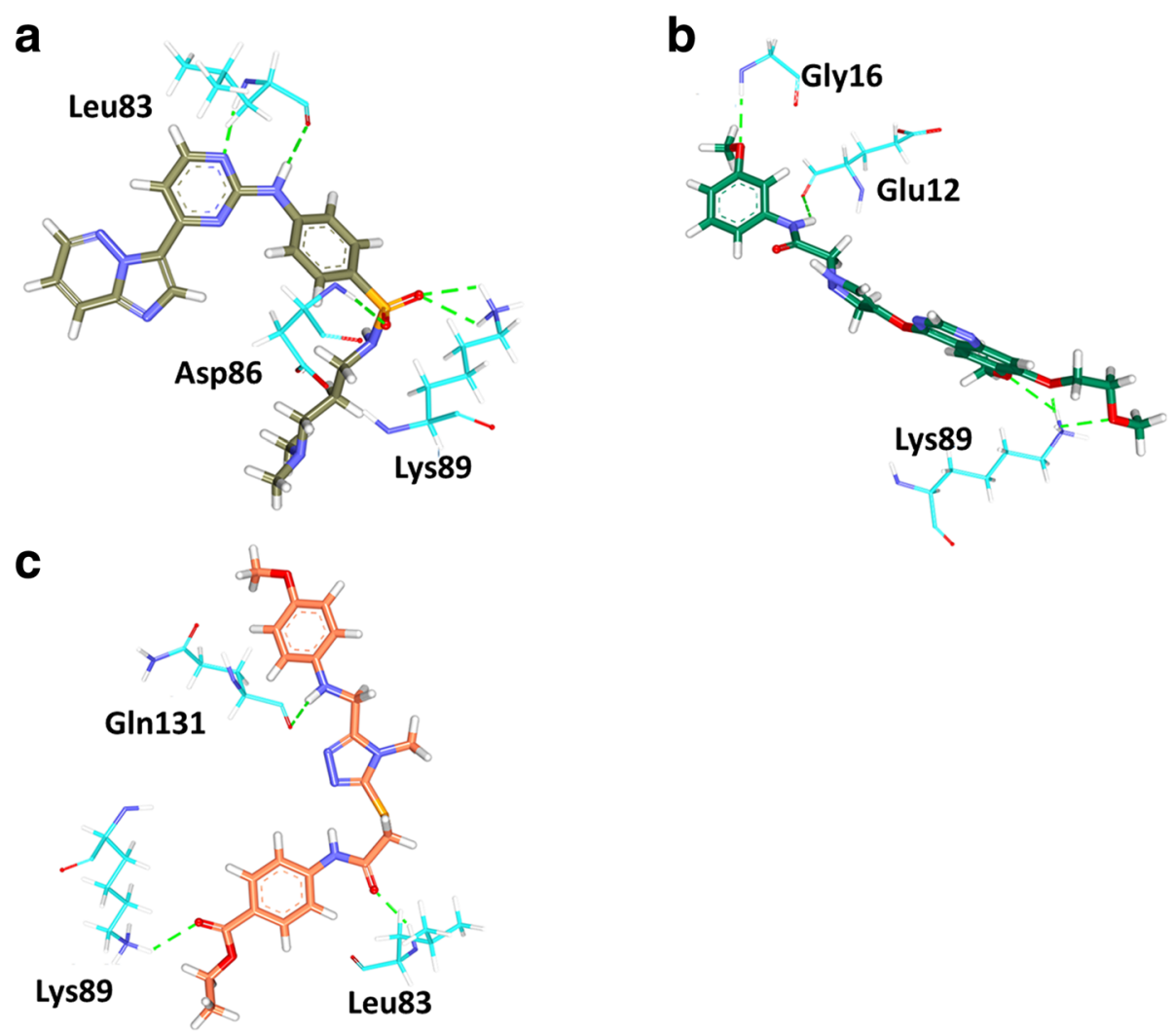

Fig. 19 Intermolecular interactions between the ligand and the protein. The co-crystal is represented in gray, reference is denoted in green and the Hit in orange. Green dotted lines represent the hydrogen bonds. The protein residues are indicated in cyan

Therefore, the presence pentene ring in the ligand apparently seems to be important in making this inhibitor of a type II class. Additionally, the sulphur group of Cys1045 has involved with the pentane ring. Additionally, the first benzene ring was held by Leu840 and Leu1035 and the second benzene ring was held by Leu889. Furthermore, it can be observed that the bulky groups present within the ligand have been held by either hydrogen bonds or the hydrophobic bonds, thus providing proper positioning for the ligand.

Furthermore, we challenged the identified Hit against CDK2 target whose inhibition can abrupt the progression of the cancer cells. The CDK2 displays four binding sites such as ATP binding site (competitive) and two non- competitive sites. Additionally, upon subjecting to the cyclin binding process gives rise to a new site caused

Table 6 Intermolecular interaction between the protein and the ligands

\begin{tabular}{|c|c|c|c|c|c|}
\hline \multirow{2}{*}{$\begin{array}{l}\text { Name of the } \\
\text { compound }\end{array}$} & \multicolumn{3}{|c|}{ Hydrogen bonds $<3 \AA$} & \multirow[t]{2}{*}{ van der Waals interactions } & \multirow[t]{2}{*}{$\pi-\pi$} \\
\hline & Residue atom & Ligand atom & Bond length & & \\
\hline Co-crystal & $\begin{array}{l}\text { Leu83: O } \\
\text { Leu83:HN } \\
\text { Asp86:HN } \\
\text { Lys89:HZ2 } \\
\text { Lys89:HZ3 }\end{array}$ & $\begin{array}{l}\mathrm{H} 42 \\
\mathrm{~N} 20 \\
\mathrm{O} 31 \\
\mathrm{O} 32 \\
\mathrm{O} 32\end{array}$ & $\begin{array}{l}2.2 \\
2.0 \\
2.5 \\
2.4 \\
2.4\end{array}$ & $\begin{array}{l}\text { Gly11, Glu12, Lys20, Lys33, Val64, } \\
\text { Glu81, Gln85, Lys88, Gln131, } \\
\text { Asn132, Leu148, }\end{array}$ & $\begin{array}{l}\text { Val18, Ala31, Phe80, Phe82, } \\
\text { Leu134, Ala144 }\end{array}$ \\
\hline Reference & $\begin{array}{l}\text { Glu12: O } \\
\text { Gly16:HN } \\
\text { Lys89:HZ2 } \\
\text { Lys89:HZ2 } \\
\text { Lys89:HZ3 }\end{array}$ & $\begin{array}{l}\mathrm{H} 43 \\
\mathrm{O} 27 \\
\mathrm{O} 34 \\
\mathrm{O} 31 \\
\mathrm{O} 29\end{array}$ & $\begin{array}{l}3.0 \\
2.2 \\
2.3 \\
1.9 \\
2.5\end{array}$ & $\begin{array}{l}\text { Gly11, Tyr15, Val18, Gln85, Asp86, } \\
\text { Asp127, Lys129, Gln131, Asn132, } \\
\text { Leu134, Leu298, }\end{array}$ & Phe82 \\
\hline Hit & $\begin{array}{l}\text { Leu83:HN } \\
\text { Lys89:HZ2 } \\
\text { Gln131: O }\end{array}$ & $\begin{array}{l}\mathrm{O} 25 \\
\mathrm{O} 24 \\
\mathrm{H} 36\end{array}$ & $\begin{array}{l}2.1 \\
2.4 \\
2.2\end{array}$ & $\begin{array}{l}\text { Gly11, Glu12, Ala31, Lys33, Val64, } \\
\text { Phe82, His84, GIn85, Asp86, Lys129, } \\
\text { Asn132, Asp145, Ala144, Val164 }\end{array}$ & Ile10, Val18, Leu134, Leu298 \\
\hline
\end{tabular}




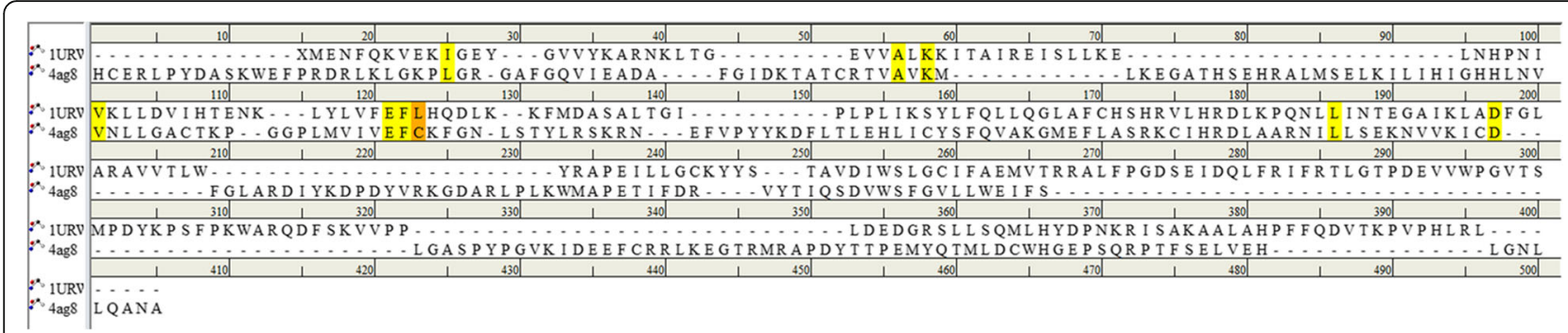

Fig. 20 Sequence alignment of the protein targets representing angiogenesis (4AG8) and progression (1URW). The sequence identity and the sequence similarity were found to be 20.1 and 38.1, respectively. The active site shows higher degree of identity as demonstrated by the residues highlighted in yellow. The orange amino acids refer to the key inhibitory residues found to be complementary with each other

due to the conformational changes in the ATP site and is labeled as the allosteric site (site IV). Amongst them the ATP binding site is highly conserved across all the human kinases [23]. Therefore, we focused on the ATP binding site to evaluate the potency of the identified ligand. This ATP binding site is a cleft located between the $\mathrm{N}$-terminal domain consisting of beta-sheets (small lobe) and a C-terminal domain rich in helices (large lobe) [71], which can be further divided into three. The first one is the hydrophobic region comprising of Ile10, Ala31, Val64, Phe80, Glu81, Phe82, Leu83, Leu134 and Ala144. The second region consists of three amino acids, Val18, Asp86 and Gln131 and generally interacts with the ribose moiety of the ATP. The third region is made up of Lys33, Asp145 and Asn132. Additionally, two domains are joined by the hinge region constituting Glu81 and Leu83, respectively. The identified ligand has formed an hydrogen bond interactions with the key residues involving the Leu83 and Lys89 [65, 71]. An additional hydrogen bond was formed with the amino acid Gln131 that is located at the polar interaction site through its $\mathrm{HN}$ group. This pattern of binding is in agreement as described previously [71]. The residues belonging to the hydrophobic region consisting of Ile10, Val18 and Leu134 have joined with the ligand by the hydrophobic bonds. The residues, Val64, Ala144 of the hydrophobic interaction site and Lys33, Asp86 and Asp145 that belong to the polar interaction site have interacted with the ligand through the van der Waal's interactions and thus facilitating the lead molecule to be

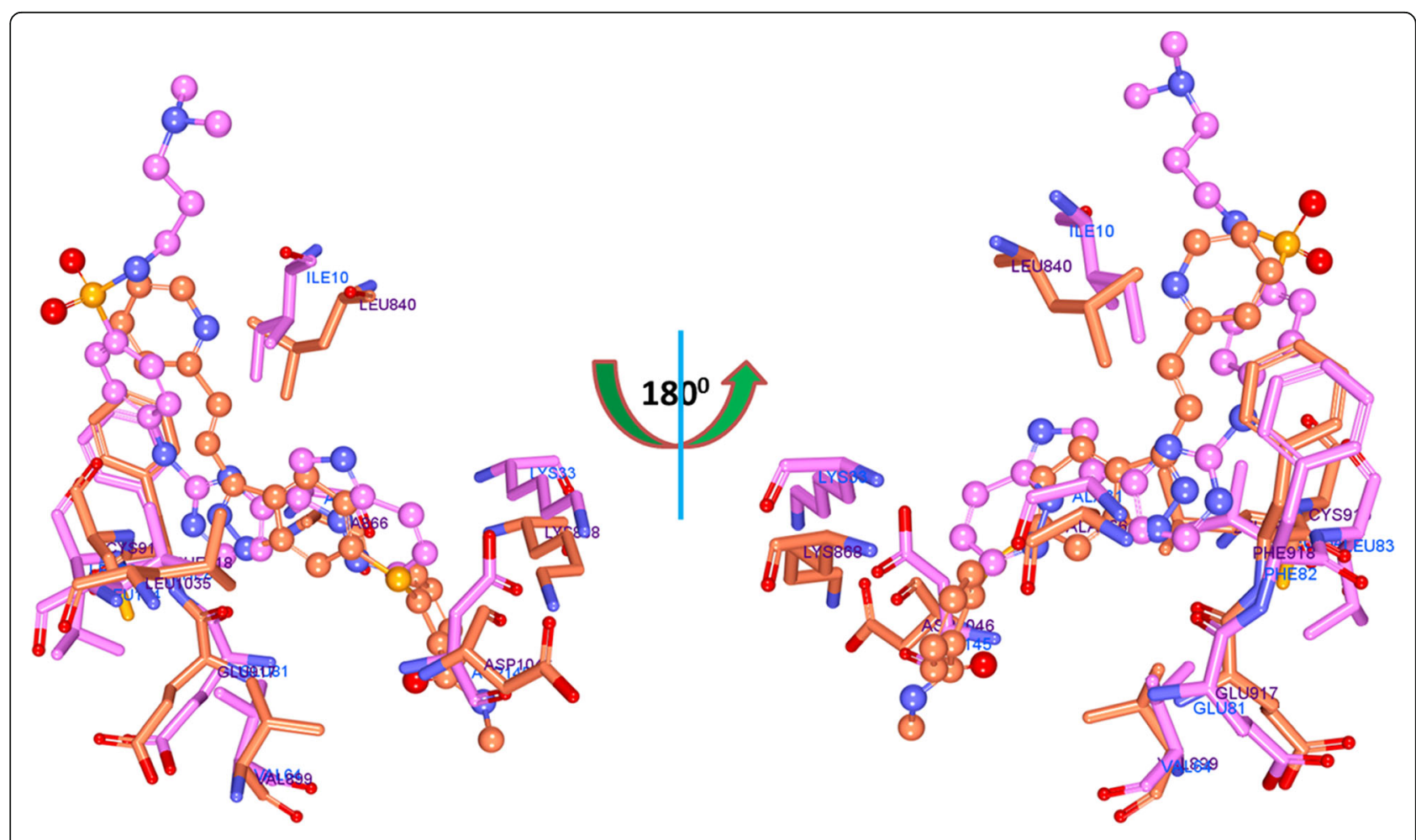

Fig. 21 Comparison of the active sites of 4AG8 (orange) and 1URW (pink). The corresponding ligand molecule is represented in ball and stick model 
seated firmly with in the active site. Furthermore, since the ATP binding sites are conserved across the kinases [71], this could be one reason for the complementarity that exists between Cyc919 of VEGFR2 and Leu83 of CDK2, (Fig. 20).

\section{Conclusions}

The present study aims at identifying a dual inhibitor that can repress the angiogenesis and progression. In order to achieve this, a systematic ligand-based pharmacophore modelling and subsequent large database screening was conducted. From the obtained lead molecules, further knowledge based screening was conducted to find the compounds that obeyed the back-to-front type of inhibitory mechanism employing the docking protocol and the interactions with the key residues. Consequently, results showed that only one compound has qualified this criterion. Upon further studies and applying the MD simulations, it was observed that the ligand-protein complex was relatively stable. Therefore this lead compound was challenged against 1URW, where the Hit was found to interact with the important residues seated at its active site. Our results also demonstrate that binding with Cys919 and Lue83 are important in designing a dual inhibitor for angiogenesis and progression. Taken together, we suggest that the Hit compound might be a potential lead candidate that can repress cancer angiogenesis and growth.

\section{Additional files}

Additional file 1: Test set assessment. Assessing the test set values for estimated and the experimental activities by Hypo1. (DOCX $18 \mathrm{~kb}$ )

Additional file 2: Cocrystal re-dock results of 4AG8. Overlapping of the co-crystal (cyan) onto the docked pose (orange). The binding pattern was found to be similar. (DOCX $181 \mathrm{~kb}$ )

Additional file 3: $2 \mathrm{D}$ interaction representation of the the reference compound and 4AG8. Detailed molecular interactions of the reference compound. (DOCX $420 \mathrm{~kb}$ )

Additional file 4: $2 D$ interaction representation of the Hit compound and 4AG8. Detailed molecular interactions of the Hit compound. (DOCX $424 \mathrm{~kb}$ )

Additional file 5: Docking of the co-crystal within the binding pocket of 1 URW. Docking of the co-crystal within the binding pocket. Pink is the docked pose and green represents the co-crystal position. (DOCX $142 \mathrm{~kb}$ )

Additional file 6: $2 \mathrm{D}$ interaction representation of the co-crystal and 1 URW. Detailed molecular interactions of the co-crystal compound. (DOCX 229 kb)

Additional file 7: $2 \mathrm{D}$ interaction representation of the reference compound and 1 URW. Molecular interaction details of the reference compound. (DOCX $204 \mathrm{~kb}$ )

Additional file 8: $2 \mathrm{D}$ interaction representation of the Hit compound and 1URW. Molecular interaction details of the Hit compound. (DOCX 264 kb)

Additional file 9: Active sites comparison. Comparison of the active site residues of $4 A G 8$ and 1 UMR. (DOCX $13 \mathrm{~kb}$ )

\section{Abbreviations}

2D: Two Dimensiional; 3D QSAR: Three-dimensional quantitative structureactivity relationships; 3D: Three Dimensional; $\AA$ : Angstrom; A: Number of actives; ACD: Advanced chemistry development; ADMET: Absorption, distribution, metabolism, excretion, toxicity; ATP: Adenosine Triphosphate; B3LYP: Becke, 3-parameter, Lee-Yang-Parr; BBB: Blood Brain Barrier; CDK: Cyclin-dependent kinases; CHARMm: Chemistry at HARvard Macromolecular Mechanics; CYPs: Cytochromes P450; D: Total number of molecules in a database; DFT: Density functional theory; DGF: Asp-Phe-Gly; DS: Discovery Studio; EF: Enrichment Factor; Ff: force field; G1: Gap 1; GF: Goodness of Fit; GOLD: Genetic optimisation for ligand docking; GROMACS: GROningen machine for chemical simulations; Ha: Total Actives; HBA: Hydrogen bond acceptor; HBD: Hydrogen bond donor; HIA: Human intestinal absorption; HOMO: Highest occupied Molecular Orbitals; Ht: Total Hits; Hy Ali: Hydrophobic aliphatic; HyP: HydroPhobic; IC 50 : Half maximal inhibitory concentration; LINCS: Linear constraint solver for molecular simulations; LUMO: Lowest unoccupied molecular orbitals; Max: Maximum; MD: Molecular dynamics; NCl: National cancer institute; NPT: Number of particles, pressure, and temperature; NVT: Number of particles, volume, and temperature; PDB: Protein data bank; PM: Pocket match; PME: Patricle mesh ewald; PPB: Plasma protein binding; RA: Ring aromatic; RKT: Receptor Tyrosine Kinases; RMSD: Root mean square deviation; S phase: Synthesis phase; TIP3P: Three-site transferrable intermolecular potential; VEGFRs: Vascular endothelial growth factor receptors; VEGFs: Vascular endothelial growth factors; VMD: Visual molecular dynamics

\section{Acknowledgements}

Not applicable

\section{Funding}

This research was supported by Next-Generation BioGreen 21 Program from Rural Development administration, Republic of Korea (Grant no. PJ01106202), and also supported by the Pioneer research Center Program through the National Research Foundation (NRF) funded by the Ministry o of Science, ICT and Future Planning (NRF-2015M3C1A3023028).

\section{Availability of data and materials}

All the data and the material are provided with the manuscript and the Additional files 1, 2, 3, 4, 5 and 6 .

Authors' contributions

$K W L, S R$ and $A B$ designed the project. SR and $A Z$ wrote the manuscript. SR and $\mathrm{KWL}$ assessed the results. All the authors read and approve the manuscript.

Ethics approval and consent to participate

Not applicable

\section{Consent for publication}

Not applicable

\section{Competing interests}

The authors declare that they have no competing interests

\section{Publisher's Note}

Springer Nature remains neutral with regard to jurisdictional claims in published maps and institutional affiliations.

Received: 23 October 2017 Accepted: 24 January 2018

Published online: 07 March 2018

References

1. Nishida N, Yano H, Nishida T, Kamura T, Kojiro M. Angiogenesis in cancer. Vasc Health Risk Manag. 2006;2(3):213-9.

2. Ucuzian AA, Gassman AA, East AT, Greisler HP. Molecular mediators of angiogenesis. J Burn Care Res. 2010;31(1):158-75.

3. Shibuya M. Vascular endothelial growth factor (VEGF) and its receptor (VEGFR) signaling in angiogenesis: a crucial target for anti- and proAngiogenic therapies. Genes Cancer. 2011;2(12):1097-105.

4. Koch S, Claesson-Welsh L. Signal transduction by vascular endothelial growth factor receptors. Cold Spring Harb Perspect Med. 2012;2(7):a006502. 
5. Li J, Zhou N, Luo K, Zhang W, Li X, Wu C, Bao J. In silico discovery of potential VEGFR-2 inhibitors from natural derivatives for anti-angiogenesis therapy. Int J Mol Sci. 2014;15(9):15994-6011.

6. Han KY, Dugas-Ford J, Lee H, Chang JH, Azar DT. MMP14 cleavage of VEGFR1 in the cornea leads to a VEGF-trap Antiangiogenic effect. Invest Ophthalmol Vis Sci. 2015;56(9):5450-6.

7. Rahimi N. VEGFR-1 and VEGFR-2: two non-identical twins with a unique physiognomy. Front Biosci. 2006;11:818-29.

8. Witmer AN, van Blijswijk BC, Dai J, Hofman P, Partanen TA, Vrensen GF, Schlingemann RO. VEGFR-3 in adult angiogenesis. J Pathol. 2001;195(4):490-7.

9. Taylor PC. VEGF and imaging of vessels in rheumatoid arthritis. Arthritis Res. 2002;4(Suppl 3):S99-107.

10. Fava GA. Affective disorders and endocrine disease. New insights from psychosomatic studies. Psychosomatics. 1994;35(4):341-53.

11. Detmar M. The role of VEGF and thrombospondins in skin angiogenesis. J Dermatol Sci. 2000;24(Suppl 1):S78-84.

12. Lee YJ, Karl DL, Maduekwe UN, Rothrock C, Ryeom S, D'Amore PA, Yoon SS. Differential effects of VEGFR-1 and VEGFR-2 inhibition on tumor metastases based on host organ environment. Cancer Res. 2010;70(21):8357-67.

13. Homayouni M. Vascular endothelial growth factors and their inhibitors in ocular Neovascular disorders. J Ophthalmic Vis Res. 2009;4(2):105-14.

14. Hsu KC, Sung TY, Lin CT, Chiu YY, Hsu JT, Hung HC, Sun CM, Barve I, Chen WL, Huang WC, et al. Anchor-based classification and type-C inhibitors for tyrosine kinases. Sci Rep. 2015;5:10938.

15. Dar AC, Shokat KM. The evolution of protein kinase inhibitors from antagonists to agonists of cellular signaling. Annu Rev Biochem. 2011;80: 769-95.

16. Fedorov O, Muller S, Knapp S. The (un) targeted cancer kinome. Nat Chem Biol. 2010;6(3):166-9.

17. Sanphanya K, Wattanapitayakul SK, Phowichit S, Fokin W, Vajragupta O. Novel VEGFR-2 kinase inhibitors identified by the back-to-front approach. Bioorg Med Chem Lett. 2013;23(10):2962-7.

18. Honda T, Nagahara H, Mogi H, Ban M, Aono H. KDR inhibitor with the intramolecular non-bonded interaction: conformation-activity relationships of novel indole-3-carboxamide derivatives. Bioorg Med Chem Lett. 2011; 21(6):1782-5.

19. Bauer D, Whittington DA, Coxon A, Bready J, Harriman SP, Patel VF, Polverino A, Harmange JC. Evaluation of indazole-based compounds as a new class of potent KDRNEGFR-2 inhibitors. Bioorg Med Chem Lett. 2008; 18(17):4844-8.

20. Jiang P, Du W, Wu M. Regulation of the pentose phosphate pathway in cancer. Protein Cell. 2014;5(8):592-602.

21. Malumbres M. Cyclin-dependent kinases. Genome Biol. 2014;15(6):122.

22. Neganova I, Lako M. G1 to S phase cell cycle transition in somatic and embryonic stem cells. J Anat. 2008;213(1):30-44.

23. Li Y, Zhang J, Gao W, Zhang L, Pan Y, Zhang S, Wang Y. Insights on structural characteristics and Ligand binding mechanisms of CDK2. Int J Mo Sci. 2015;16(5):9314-40.

24. Sherr CJ. G1 phase progression: cycling on cue. Cell. 1994;79(4):551-5.

25. Chen Y-NP, Sharma SK, Ramsey TM, Jiang L, Martin MS, Baker K, Adams PD, Bair KW, Kaelin WG. Selective killing of transformed cells by cyclin/cyclindependent kinase 2 antagonists. Proc Natl Acad Sci U S A. 1999;96(8):4325-9.

26. Latham AM, Kankanala J, Fearnley GW, Gage MC, Kearney MT, HomerVanniasinkam S, Wheatcroft SB, Fishwick CW, Ponnambalam S. In silico design and biological evaluation of a dual specificity kinase inhibitor targeting cell cycle progression and angiogenesis. PLoS One. 2014;9(11): e110997.

27. Baldwin I, Bamborough P, Haslam CG, Hunjan SS, Longstaff T, Mooney CJ, Patel S, Quinn J, Somers DO. Kinase array design, back to front: biaryl amides. Bioorg Med Chem Lett. 2008;18(19):5285-9.

28. Iwata H, Oki H, Okada K, Takagi T, Tawada M, Miyazaki Y, Imamura S, Hori A, Lawson JD, Hixon MS, et al. A back-to-front fragment-based drug design search strategy targeting the DFG-out pocket of protein tyrosine Kinases. ACS Medl Chem Lett. 2012;3(4):342-6.

29. Regan J, Breitfelder S, Cirillo P, Gilmore T, Graham AG, Hickey E, Klaus B, Madwed J, Moriak M, Moss N, et al. Pyrazole urea-based inhibitors of p38 MAP kinase: from lead compound to clinical candidate. J Med Chem. 2002; 45(14):2994-3008.

30. Zhang Y, Yang S, Jiao Y, Liu H, Yuan H, Lu S, Ran T, Yao S, Ke Z, Xu J, et al. An integrated virtual screening approach for VEGFR-2 inhibitors. J Chem Inf Mod. 2013;53(12):3163-77.
31. Kawakami JK, Martinez Y, Sasaki B, Harris M, Kurata WE, Lau AF. Investigation of a novel molecular descriptor for the lead optimization of 4-aminoquinazolines as vascular endothelial growth factor receptor-2 inhibitors: application for quantitative structure-activity relationship analysis in lead optimization. Bioorg Med Chem Lett. 2011;21(5):1371-5.

32. Potashman MH, Bready J, Coxon A, DeMelfi TM Jr, DiPietro L, Doerr N, Elbaum D, Estrada J, Gallant P, Germain J, et al. Design, synthesis, and evaluation of orally active benzimidazoles and benzoxazoles as vascular endothelial growth factor-2 receptor tyrosine kinase inhibitors. J Med Chem. 2007;50(18):4351-73.

33. Zhang L, Zheng Q, Yang Y, Zhou H, Gong X, Zhao S, Fan C. Synthesis and in vivo SAR study of indolin-2-one-based multi-targeted inhibitors as potential anticancer agents. Eur J Med Chem. 2014;82:139-51.

34. Caballero J, Munoz C, Alzate-Morales JH, Cunha S, Gano L, Bergmann R, Steinbach J, Kniess T. Synthesis, in silico, in vitro, and in vivo investigation of 5-[(1) (1)C] methoxy-substituted sunitinib, a tyrosine kinase inhibitor of VEGFR-2. Eur J Med Chem. 2012;58:272-80.

35. Kuchar M, Oliveira MC, Gano L, Santos I, Kniess T. Radioiodinated sunitinib as a potential radiotracer for imaging angiogenesis-radiosynthesis and first radiopharmacological evaluation of 5-[125l] lodo-sunitinib. Bioorg Med Chem Lett. 2012;22(8):2850-5.

36. Bhide RS, Cai ZW, Zhang YZ, Qian L, Wei D, Barbosa S, Lombardo L, Borzilleri RM, Zheng X, Wu LI, et al. Discovery and preclinical studies of (R)1-(4-(4-fluoro-2-methyl-1H-indol-5-yloxy)-5- methylpyrrolo [2,1-f] [1,2,4] triazin-6-yloxy) propan-2-ol (BMS-540215), an in vivo active potent VEGFR-2 inhibitor. J Med Chem. 2006;49(7):2143-6.

37. Wang S, Midgley CA, Scaerou F, Grabarek JB, Griffiths G, Jackson W, Kontopidis G, McClue SJ, Mclnnes C, Meades C, et al. Discovery of N-phenyl-4-(thiazol-5-yl) pyrimidin-2-amine aurora kinase inhibitors. J Med Chem. 2010;53(11):4367-78.

38. Kiselyov AS, Semenova M, Semenov W. 3,4-Disubstituted isothiazoles: novel potent inhibitors of VEGF receptors 1 and 2. Bioorg Med Chem Lett. 2009; 19(4):1195-8.

39. Iwata H, Imamura S, Hori A, Hixon MS, Kimura H, Miki H. Biochemical characterization of a novel type-II VEGFR2 kinase inhibitor: comparison of binding to non-phosphorylated and phosphorylated VEGFR2. Bioorg Med Chem. 2011;19(18):5342-51.

40. Sakkiah S, Thangapandian S, John S, Kwon YJ, Lee KW. 3D QSAR pharmacophore based virtual screening and molecular docking for identification of potential HSP90 inhibitors. Eur J Med Chem. 2010;45(6):2132-40.

41. Debnath AK. Pharmacophore mapping of a series of 2,4-diamino-5deazapteridine inhibitors of Mycobacterium Avium Complex dihydrofolate reductase. J Med Chem. 2002;45(1):41-53.

42. Leeson PD, Davis AM, Steele J. Drug-like properties: guiding principles for design - or chemical prejudice? Drug Discov Today Techs. 2004;1 (3):189-95.

43. Lipinski CA, Lombardo F, Dominy BW, Feeney PJ. Experimental and computational approaches to estimate solubility and permeability in drug discovery and development settings. Adv Drug Del Rev. 2001;46(1-3):3-26.

44. Jones G, Willett P, Glen RC, Leach AR, Taylor R. Development and validation of a genetic algorithm for flexible docking. J Mol Biol. 1997;267(3):727-48.

45. Verdonk ML, Cole JC, Hartshorn MJ, Murray CW, Taylor RD. Improved proteinligand docking using GOLD. Proteins Struct Funct Bioinform. 2003;52(4):609-23.

46. Vardhini SRD. In silico evaluation for the potential naturally available drugs for breast cancer. J Recept Signal Transduct. 2014;34(3):174-9.

47. Kavitha R, Karunagaran S, Chandrabose SS, Lee KW, Meganathan C. Pharmacophore modeling, virtual screening, molecular docking studies and density functional theory approaches to identify novel ketohexokinase (KHK) inhibitors. Biosystems. 2015;138:39-52.

48. Rampogu S, Baek A, Son M, Zeb A, Park C, Kumar R, Lee G, Kim D, Choi Y, Cho Y, et al. Computational exploration for lead compounds that can reverse the nuclear morphology in Progeria. Biomed Res Int. 2017;2017:15.

49. Zoete V, Cuendet MA, Grosdidier A, Michielin O. SwissParam: a fast force field generation tool for small organic molecules. J Comput Chem. 2011; 32(11):2359-68.

50. Rampogu S, Son M, Park C, Kim H-H, Suh J-K, Lee KW. Sulfonanilide derivatives in identifying novel Aromatase inhibitors by applying docking, virtual screening, and MD simulations studies. Biomed Res Int. 2017;2017:17.

51. Mackerell AD Jr. Empirical force fields for biological macromolecules: overview and issues. J Comput Chem. 2004;25(13):1584-604.

52. MacKerell AD, Bashford D, Bellott M, Dunbrack RL, Evanseck JD, Field MJ, Fischer S, Gao J, Guo H, Ha S, et al. All-atom empirical potential for molecular modeling and dynamics studies of proteins. J Phys Chem B. 1998;102(18):3586-616. 
53. Zhu X, Lopes PEM, MacKerell AD. Recent developments and applications of the CHARMM force fields. Wiley Interdiscip Rev Comput Mol Sci. 2012;2(1):167-85.

54. Mallajosyula SS, Jo S, Im W, Mackerell AD Jr. Molecular dynamics simulations of glycoproteins using CHARMM. Methods Mol Biol (Clifton, NJ). 2015;1273: 407-29.

55. Van Der Spoel D, Lindahl E, Hess B, Groenhof G, Mark AE, Berendsen HJ. GROMACS: fast, flexible, and free. J Comput Chem. 2005;26(16):1701-18.

56. Bavi R, Kumar R, Rampogu S, Son M, Park C, Baek A, Kim HH, Suh JK, Park SJ, Lee KW. Molecular interactions of UvrB protein and DNA from helicobacter pylori: insight into a molecular modeling approach. Comput Biol Med. 2016; 75:181-9.

57. Berendsen HJC, Postma JPM, WFv G, DiNola A, Haak JR. Molecular dynamics with coupling to an external bath. J Chem Phys. 1984;81(8):3684-90.

58. Parrinello M, Rahman A. Polymorphic transitions in single crystals: a new molecular dynamics method. J Appl Phys. 1981:52(12):7182-90.

59. Hess B, Bekker H, Berendsen HJC, Fraaije JGEM. LINCS: a linear constraint solver for molecular simulations. J Comput Chem. 1997;18(12):1463-72.

60. Darden T, York D, Pedersen L. Particle mesh Ewald: an N.log(N) method for Ewald sums in large systems. J Chem Phys. 1993;98(12):10089-92.

61. Humphrey W, Dalke A, Schulten K. VMD: visual molecular dynamics. J Mol Graph. 1996;14(1):33-8.

62. Banavath HN, Sharma OP, Kumar MS, Baskaran R. Identification of novel tyrosine kinase inhibitors for drug resistant T315I mutant BCR-ABL: a virtual screening and molecular dynamics simulations study. Sci Rep. 2014;4:6948.

63. Mihara M, Shintani S, Nakahara Y, Kiyota A, Ueyama Y, Matsumura T, Wong DT. Overexpression of CDK2 is a prognostic indicator of oral cancer progression. Jpn J Cancer Res Gann. 2001;92(3):352-60.

64. Deshpande A, Sicinski P, Hinds PW. Cyclins and cdks in development and cancer: a perspective. Oncogene. 2005;24(17):2909-15.

65. Byth KF, Cooper N, Culshaw JD, Heaton DW, Oakes SE, Minshull CA, Norman RA, Pauptit RA, Tucker JA, Breed J, et al. Imidazo [1,2-b] pyridazines: a potent and selective class of cyclin-dependent kinase inhibitors. Bioorg Med Chem Lett. 2004;14(9):2249-52.

66. Yeturu K, Chandra N. PocketMatch: a new algorithm to compare binding sites in protein structures. BMC Bioinform. 2008;9(1):543.

67. Zwick E, Bange J, Ullrich A. Receptor tyrosine kinases as targets for anticancer drugs. Trends Mol Med. 2002;8(1):17-23.

68. Folkman J. Role of angiogenesis in tumor growth and metastasis. Semin Oncol. 2002;29(6 Suppl 16):15-8.

69. Hoeben A, Landuyt B, Highley MS, Wildiers H, Van Oosterom AT, De Bruijn EA. Vascular endothelial growth factor and angiogenesis. Pharmacoll Rev. 2004;56(4):549-80.

70. Schenone S, Bondavalli F, Botta M. Antiangiogenic agents: an update on small molecule VEGFR inhibitors. Curr Med Chem. 2007:14(23):2495-516.

71. Tripathi SK, Muttineni R, Singh SK. Extra precision docking, free energy calculation and molecular dynamics simulation studies of CDK2 inhibitors. J Theor Biol. 2013;334:87-100.

\section{Submit your next manuscript to BioMed Central and we will help you at every step:}

- We accept pre-submission inquiries

- Our selector tool helps you to find the most relevant journal

- We provide round the clock customer support

- Convenient online submission

- Thorough peer review

- Inclusion in PubMed and all major indexing services

- Maximum visibility for your research

Submit your manuscript at www.biomedcentral.com/submit
Biomed Central 